\title{
Polynomial estimates for c-functions on reductive symmetric spaces
}

\author{
E. P. van den Ban and H. Schlichtkrull
}

$1 / 11-2010$

\begin{abstract}
The c-functions, related to a reductive symmetric space $G / H$ and a fixed representation $\tau$ of a maximal compact subgroup $K$ of $G$, are shown to satisfy polynomial bounds in imaginary directions.
\end{abstract}

\section{Introduction}

Let $X=G / H$ be a reductive symmetric space and $K \subset G$ a maximal compact subgroup. The harmonic analysis for $K$-finite functions on $G / H$ has been developed in the past 3 decades (see for example [13], [19], [11, [12, [7, 8], [9] and further references mentioned there) as a generalization of the work of HarishChandra [14. Associated to each finite dimensional representation $\left(\tau, V_{\tau}\right)$ of $K$, and to each cuspidal $\sigma$-parabolic subgroup $P$ of $G$, there is a fundamental family of functions on $G / H$, called Eisenstein integrals, on which the spectral decomposition of $L^{2}(G / H)$ is based. The asymptotic behavior of these functions, when the space variable $x \in X$ tends to infinity in various directions, is described through $c$-functions, analogous to Harish-Chandra's generalized $c$ functions. The $c$-functions, which play a profound role in the harmonic analysis, are matrix-valued meromorphi functions of the complex spectral parameter $\lambda$.

It is convenient to normalize the Eisenstein integrals so that the $c$-function corresponding to a particular asymptotic direction is 1 . The asymptotic behavior of the normalized Eisenstein integral is then described by the normalized $c$-functions, which are quotients of the unnormalized $c$-functions. They are denoted $C^{\circ}(s: \lambda)$. In this paper we shall establish a polynomial bound for these normalized $c$-functions, when $\lambda$ tends to infinity in imaginary directions, for the case where the parabolic subgroup $P$ is $\sigma$-minimal. More precisely we prove that if $\lambda$ varies such that its real part stays bounded, then there exists a polynomial $q(\lambda)$ such that the entries of the product $q(\lambda) C^{\circ}(s: \lambda)$ are polynomially bounded in $\lambda$. Obviously, the polynomial $q$ serves to eliminate singularities. Our result includes the information about this polynomial that it is a product of first order polynomials given by roots.

The result which we shall prove was stated without proof as Theorem 10.1 in [9. It was then used to obtain a characterization of the Paley-Wiener space for $G / H$, which differs from the one established in [9], and which had previously 
been conjectured in [5]. The essential difference between the two characterizations is that in [5] the growth conditions are only required in a single Weyl chamber. Every Fourier transform $\varphi$ of a compactly supported function of type $\tau$ satisfies the transformation property $\varphi(s \lambda)=C^{\circ}(s: \lambda) \varphi(\lambda)$ for each element $s$ in the Weyl group. It follows from the polynomial estimate for $C^{\circ}(s: \lambda)$ that the growth estimates of Paley-Wiener type hold for all $\lambda$ if they hold on a single chamber.

In the special case of a Riemannian symmetric space and the trivial $K$-type, the $c$-function is explicitly known from the formula of Gindikin and Karpelevic, by which it is expressed as a product of quotients of gamma functions (see for example [16], p. 447). In this case our normalized $c$-functions are the quotients $c(s \lambda) / c(\lambda)$, where $s$ belongs to the Weyl group, and the polynomial estimate follows easily from known properties of the gamma function. More generally, when the group $G$ is considered as a symmetric space for $G \times G$, a similar argument can be carried out for the quotients which normalize Harish-Chandra's generalized $c$-functions for this case. Here one employs Wallach's result [22], that the matrix entries of the generalized $c$-functions are quotients of gamma functions.

As in Harish-Chandra's case, the $c$-functions for a $K$-type $\tau$ on a reductive symmetric space $G / H$ are closely related to standard intertwining operators for the principal series. These operators satisfy estimates of polynomial type. On the subspace of $K$-finite vectors this follows for example from [17, and in general it follows from the functional equation of Vogan and Wallach [21]. In the first part of the paper, Section 1, we establish an estimate of this type for standard intertwining operators, with refined information about the polynomial factor that governs singularities. The estimate is derived by using the functional equation of [21]. As we believe this result to be of independent interest, we have written the exposition of this part without reference to the theory of reductive symmetric spaces.

The main result in the article, the estimate of $c$-functions, is stated in Thm. 2.1 in Section 2, In this section we also introduce the necessary background notation related to reductive symmetric spaces. The proof is given in Sections 3 5. The main ingredients are the previous estimate for the standard intertwining operators, a similar estimate for a map which parametrizes $H$-fixed distribution vectors in the minimal principal series of $G / H$, and finally a similar estimate for the inverse of this parametrizing map, the so-called evaluation map.

\section{Estimates for intertwining operators}

Let $G$ be a real reductive group of the Harish-Chandra class, see [20, and let $K$ be a maximal compact subgroup of $G$, with associated Cartan involution $\theta$. The differential of this involution is an involution of the Lie algebra $\mathfrak{g}$ and denoted by the same symbol. As usual, we adopt the convention to denote Lie groups by Roman capital letters, and the associated Lie algebras by the corresponding German lower case letters. Let $\mathfrak{g}=\mathfrak{k} \oplus \mathfrak{p}$ be the decomposition into a direct sum of the +1 and -1 eigenspaces of $\theta$. We extend the Killing 
form of $[\mathfrak{g}, \mathfrak{g}]$ to an $\operatorname{Ad}(G)$-invariant symmetric bilinear form $B$ on $\mathfrak{g}$, for which $\langle X, Y\rangle:=-B(X, \theta Y)$ is a positive definite inner product.

We assume that $\mathfrak{a}_{0}$ is a maximal abelian subspace of $\mathfrak{p}$. Let $\mathcal{P}$ be the set of parabolic subgroups of $G$ containing $A_{0}:=\exp \mathfrak{a}_{0}$. Then each $P \in \mathcal{P}$ has a Langlands decomposition of the form $P=M_{P} A_{P} N_{P}$, with $\mathfrak{a}_{P} \subset \mathfrak{a}_{0}$. If $L$ is a group of the Harish-Chandra class, we denote by $\mathcal{R}(L)$ the set of (equivalence classes of) continuous representations $\xi$ of $L$ in a Hilbert space with the following properties:

(a) $\xi$ has an infinitesimal character;

(b) the space of $C^{\infty}$-vectors for $L$ equals the space of $C^{\infty}$-vectors for a maximal compact subgroup $K_{L}$ of $L$.

Note that, in (b) we need only require identity of vector spaces, since the closed graph theorem then guarantees that the Fréchet spaces of smooth vectors are equal. As all maximal compact subgroups of $L$ are conjugate, condition (b) is equivalent to the similar condition for any fixed maximal compact subgroup of $L$. We note that all finite-dimensional irreducible representations of $L$ belong to $\mathcal{R}(L)$. Likewise, the irreducible unitary representations belong to $\mathcal{R}(L)$, see [23] p. 3.

Let $P \in \mathcal{P}$ and $\xi \in \mathcal{R}\left(M_{P}\right)$. We denote by $\mathcal{H}_{\xi}$ the Hilbert space in which $\xi$ is continuously realized, and by $\mathcal{H}_{\xi}^{\infty}$ the space of $C^{\infty}$ vectors for $M_{P}$. Let $\lambda \in \mathfrak{a}_{P \mathbb{C}}^{*}$. By $C^{\infty}(P: \xi: \lambda)$ we denote the space of $C^{\infty}$-functions $f: G \rightarrow \mathcal{H}_{\xi}$ transforming according to the rule

$$
f(\operatorname{man} x)=a^{\lambda+\rho_{P}} \xi(m) f(x),
$$

for all $x \in G$ and $(m, a, n) \in M_{P} \times A_{P} \times N_{P}$. Here $\rho_{P} \in \mathfrak{a}_{P}^{*}$ is $1 / 2$ times the trace of the adjoint action of $\mathfrak{a}_{P}$ on $\mathfrak{n}_{P}$. Observe that every map in this space has values in $\mathcal{H}_{\xi}^{\infty}$. The space $C^{\infty}(P: \xi: \lambda)$ is equipped with a Fréchet topology in the usual way. The restriction of the right regular representation to this space defines a continuous representation of $G$, which we denote by $\pi_{P, \xi, \lambda}$.

We denote by $C^{\infty}(K: \xi)$ the space of $C^{\infty}$ functions $\varphi: K \rightarrow \mathcal{H}_{\xi}$ transforming according to the rule

$$
\varphi(m k)=\xi(m) \varphi(k), \quad\left(k \in K, m \in M_{P} \cap K\right) .
$$

This space is also equipped with the usual Fréchet topology. It follows from condition (b) in the definition of $\mathcal{R}\left(M_{P}\right)$ that each function $\varphi \in C^{\infty}(K: \xi)$ has values in $\mathcal{H}_{\xi}^{\infty}$. Since $G=P K$ it is readily seen that the restriction map $\left.f \mapsto f\right|_{K}$ defines a topological linear isomorphism from $C^{\infty}(P: \xi: \lambda)$ onto $C^{\infty}(K$ : $\xi)$. Accordingly, the representation $\pi_{P, \xi, \lambda}$ may be identified with a continuous representation of $G$ in $C^{\infty}(K: \xi)$, which we shall denote by the same symbol. This is called the compact picture of the principal series representation.

The results of this section are based on the Vogan-Wallach functional equation for the standard intertwining operator. This equation has been established for a class of groups different from the Harish-Chandra class. This presents no serious complication, but we have to be somewhat careful with connected components (see the proof of Thm. 1.1). 
Let us recall the functional equation in a form that is suitable for us. If $P \in$ $\mathcal{P}$ we write $\Sigma\left(P, \mathfrak{a}_{P}\right)$ for the set of $\mathfrak{a}_{P}$-weights in $\mathfrak{n}_{P}$, and we denote by $\bar{P} \in \mathcal{P}$ the opposite parabolic subgroup with $\mathfrak{a}_{\bar{P}}=\mathfrak{a}_{P}$ and $\Sigma\left(\bar{P}, \mathfrak{a}_{\bar{P}}\right)=-\Sigma\left(P, \mathfrak{a}_{P}\right)$. If $P, Q \in \mathcal{P}$ with $\mathfrak{a}_{P}=\mathfrak{a}_{Q}$, then for $R \in \mathbb{R}$ we define the set

$$
\mathfrak{a}_{P}^{*}(Q \mid P, R):=\left\{\lambda \in \mathfrak{a}_{P \mathbb{C}}^{*} \mid\langle\operatorname{Re} \lambda, \alpha\rangle>R, \quad \forall \alpha \in \Sigma\left(\bar{Q}, \mathfrak{a}_{P}\right) \cap \Sigma\left(P, \mathfrak{a}_{P}\right)\right\} .
$$

Let now $\xi \in \mathcal{R}\left(M_{P}\right)$. Then there exists a constant $r_{\xi}>0$ such that for $f \in$ $C^{\infty}(P: \xi: \lambda)$ and $\lambda \in \mathfrak{a}_{P}^{*}\left(Q \mid P, r_{\xi}\right)$ the integral

$$
A(Q: P: \xi: \lambda) f(x)=\int_{N_{Q} \cap \bar{N}_{P}} f(n x) d n, \quad(x \in G)
$$

is absolutely convergent and defines a function in $C^{\infty}(Q: \xi: \lambda)$. Here $d n$ is a suitably normalized Haar measure on the nilpotent group $N_{Q} \cap \bar{N}_{P}$ whose precise normalization is immaterial for our discussion.

For $\lambda \in \mathfrak{a}_{P}^{*}\left(Q \mid P, r_{\xi}\right)$, the operator

$$
A(Q: P: \xi: \lambda): C^{\infty}(P: \xi: \lambda) \rightarrow C^{\infty}(Q: \xi: \lambda)
$$

is continuous linear. It intertwines the representations $\pi_{P, \xi, \lambda}$ and $\pi_{Q, \xi, \lambda}$ and is called the standard intertwining operator between these representations. Finally, for all $f \in C^{\infty}(K: \xi)$ the map $\left.\lambda \mapsto[A(Q: P: \xi: \lambda) f]\right|_{K}$ is a holomorphic $C(K: \xi)$-valued map, and there exists a constant $C>0$ such that the following estimate is valid, for all $\lambda \in \mathfrak{a}_{P}^{*}\left(Q \mid P, r_{\xi}\right)$ and all $f \in C^{\infty}(P: \xi: \lambda)$ :

$$
\|A(Q: P: \xi: \lambda) f\|_{0} \leq C\|f\|_{0} .
$$

Here $\|\cdot\|_{0}$ denotes the supremum norm over $K$ of a function $G \rightarrow \mathcal{H}_{\xi}$. All these facts are found in [21] or [23, and they are easily seen to be valid also for all groups of the Harish-Chandra class. If we combine the above estimate with the equivariance of $A(Q: P: \xi: \lambda)$ we readily infer that $\lambda \mapsto A(Q: P$ : $\xi: \lambda) f$ is a holomorphic $C^{\infty}(K: \xi)$-valued function on $\mathfrak{a}_{P}^{*}\left(Q \mid P, r_{\xi}\right)$, for every $f \in C^{\infty}(K: \xi)$.

Theorem 1.1 (Vogan-Wallach [21]) Let $P \in \mathcal{P}$ and $\xi \in \mathcal{R}\left(M_{P}\right)$. There exist non-trivial polynomial functions $b_{\xi}: \mathfrak{a}_{P \mathbb{C}}^{*} \rightarrow \mathbb{C}$ and $D_{\xi}: \mathfrak{a}_{P \mathbb{C}}^{*} \rightarrow U(\mathfrak{g})^{K}$ such that for every $f \in C^{\infty}(P: \xi: \lambda)$ and all $\lambda \in \mathfrak{a}_{P}^{*}\left(\bar{P} \mid P, r_{\xi}\right)$ the following equality is valid:

$$
\begin{aligned}
& b_{\xi}(\lambda) A(\bar{P}: P: \xi: \lambda) f \\
& \quad=A\left(\bar{P}: P: \xi: \lambda+4 \rho_{P}\right) \pi_{P, \xi, \lambda+4 \rho_{P}}\left(D_{\xi}(\lambda)\right) f .
\end{aligned}
$$

Proof. If $G$ is connected, then it is readily seen that $G$ belongs to the class of groups considered in 21]; see [10], Sect. 0.3.1, for its definition. The result then follows from [21], Thm. 1.5 (see also [23], Thm. 10.1.5).

Now assume that $G$ is a general group of the Harish-Chandra class. Then the identity component $G_{e}$ is of the Harish-Chandra class. Moreover, the representation $\xi_{e}:=\left.\xi\right|_{M_{P} \cap G_{e}}$ has the same infinitesimal character as $\xi$. The smooth 
vectors for $\xi_{e}$ are the same as those for $\xi$, hence as those for $\left.\xi\right|_{M_{P} \cap K}$, which in turn are the smooth vectors for the restriction of $\xi_{e}$ to the open subgroup $M_{P} \cap K_{e}$ of $M_{P} \cap K$. It follows that $\xi_{e} \in \mathcal{R}\left(M_{P} \cap G_{e}\right)$. Therefore, the above result is valid for the data $G_{e}, P \cap G_{e}, M_{P} \cap G_{e}$ and $\xi_{e}$. It follows that there exist polynomial maps $b_{\xi}: \mathfrak{a}_{P \mathbb{C}}^{*} \rightarrow \mathbb{C}$ and $D_{\xi}: \mathfrak{a}_{P \mathbb{C}}^{*} \rightarrow U(\mathfrak{g})^{K_{e}}$ with

$$
\begin{aligned}
& b_{\xi}(\lambda) A\left(\bar{P} \cap G_{e}: P \cap G_{e}: \xi_{e}: \lambda\right)\left(\left.f\right|_{G_{e}}\right) \\
& \quad=A\left(\bar{P} \cap G_{e}: P \cap G_{e}: \xi_{e}: \lambda+4 \rho_{P}\right) \pi_{P: G_{e}, \xi_{e}, \lambda+4 \rho_{P}}\left(D_{\xi}(\lambda)\right)\left(\left.f\right|_{G_{e}}\right)
\end{aligned}
$$

for all $f \in C^{\infty}(P: \xi: \lambda)$ and all $\lambda$ in the set $\mathfrak{a}_{P}^{*}\left(\bar{P} \mid P, r_{\xi}\right)$, where the intertwining operator on the left is given by an absolutely convergent integral. In fact, since the integrals for $G$ and $G_{e}$ are identical, we have

$$
A\left(\bar{P} \cap G_{e}: P \cap G_{e}: \xi_{e}: \lambda\right)\left(\left.f\right|_{G_{e}}\right)=\left.[A(\bar{P}: P: \xi: \lambda) f]\right|_{G_{e}}
$$

for all $\mathfrak{a}_{P}^{*}\left(\bar{P} \mid P, r_{\xi}\right)$. Similarly

$$
\begin{array}{r}
A\left(\bar{P} \cap G_{e}: P \cap G_{e}: \xi_{e}: \lambda+4 \rho_{P}\right) \pi_{P: G_{e}, \xi_{e}, \lambda+4 \rho_{P}}\left(D_{\xi}(\lambda)\right)\left(\left.f\right|_{G_{e}}\right) \\
=\left.\left[A\left(\bar{P}: P: \xi: \lambda+4 \rho_{P}\right) \pi_{P, \xi, \lambda+4 \rho_{P}}\left(D_{\xi}(\lambda)\right) f\right]\right|_{G_{e}} .
\end{array}
$$

It thus follows that

$$
\begin{aligned}
& b_{\xi}(\lambda)[A(\bar{P}: P: \xi: \lambda) f](g) \\
& \quad=\left[A\left(\bar{P}: P: \xi: \lambda+4 \rho_{P}\right) \pi_{P, \xi, \lambda+4 \rho_{P}}\left(D_{\xi}(\lambda)\right) f\right](g) .
\end{aligned}
$$

for all $g \in G_{e}$. Since $G=M_{P} G_{e}$ the identity (1.8) then follows for $g \in G$ by means of (1.1).

It only remains to be seen that (1.7) can be arranged with $D_{\xi}: \mathfrak{a}_{P \mathbb{C}}^{*} \rightarrow U(\mathfrak{g})^{K}$ instead of $D_{\xi}: \mathfrak{a}_{P \mathbb{C}}^{*} \rightarrow U(\mathfrak{g})^{K_{e}}$. Let $k \in K$. By application of (1.8) with $R_{k^{-1}} f$ and $g k$ in place of $f$ and $g$, respectively, we derive easily from the intertwining property of $A$ that

$$
\begin{aligned}
& b_{\xi}(\lambda) A(\bar{P}: P: \xi: \lambda) f \\
& \quad=A\left(\bar{P}: P: \xi: \lambda+4 \rho_{P}\right) \pi_{P, \xi, \lambda+4 \rho_{P}}\left(\operatorname{Ad}(k) D_{\xi}(\lambda)\right) f .
\end{aligned}
$$

The desired identity (1.7) now follows with $D_{\xi}(\lambda)$ replaced by the integral over $K$ of $\operatorname{Ad}(k) D_{\xi}(\lambda)$. It is easily seen that this integral is again a polynomial in $\lambda$, and it is non-trivial because the left side of (1.7) is non-trivial for some $f$.

The functional equation leads to meromorphic continuation of the standard intertwining operator with suitable estimates. We will cast these estimates in a form which is suitable for the rest of this paper. We need the following preparations.

For $s \in \mathbb{N}=\{0,1, \ldots\}$ we denote by $C^{s}(K: \xi)$ the space of $C^{s}$ functions $\varphi: K \rightarrow \mathcal{H}_{\xi}$ transforming according to the rule (1.2). As usual we write $C$ for $C^{0}$. As $K$ is compact, it follows that $C^{s}(K: \xi)$ can be equipped with a Banach norm $\|\cdot\|_{s}$ as follows. Let $X_{1}, \ldots, X_{m}$ be a basis for $\mathfrak{k}$, and let $X^{n}=X_{1}^{n_{1}} \cdots X_{m}^{n_{m}} \in \mathcal{U}(\mathfrak{k})$ for a multi-index $n \in \mathbb{N}^{m}$, then

$$
\|f\|_{s}:=\sum_{|n| \leq s}\left\|R_{X^{n}} f\right\|_{0}
$$


It is easily seen that the right regular action of $K$ on $C^{s}(K: \xi)$ is bounded with respect to this norm.

For every $s \in \mathbb{N}$, it follows from (1.6) and the intertwining property of $A(Q: P: \xi: \lambda)$ that there exists a constant $C>0$ such that the following estimate is valid, for all $f \in C^{\infty}(K: \xi)$ and $\lambda \in \mathfrak{a}_{P}^{*}\left(Q \mid P, r_{\xi}\right)$

$$
\|A(Q: P: \xi: \lambda) f\|_{s} \leq C\|f\|_{s} .
$$

It follows that the intertwining operator extends to a bounded linear endomorphism of the Banach space $C^{s}(K: \xi)$, for $\lambda$ in the indicated region. Moreover, the operator norm of this extension is uniformly bounded in $\lambda$. By an easy application of the Cauchy integral formula, it follows that the intertwining operator is holomorphic in the variable $\lambda \in \mathfrak{a}_{P}^{*}\left(Q \mid P, r_{\xi}\right)$, as a function with values in the Banach space $B\left(C^{s}(K: \xi)\right)$ of bounded linear endomorphisms, equipped with the operator norm.

Lemma 1.2 Let $D \in U(\mathfrak{g})$ be an element of order at most $d$. Then there exists a constant $t \in \mathbb{N}$, and for every $s \in \mathbb{N}$ a constant $C>0$, such that

$$
\left\|\pi_{P, \xi, \lambda}(D) f\right\|_{s} \leq C(1+|\lambda|)^{d}\|f\|_{s+t},
$$

for all $f \in C^{\infty}(K: \xi)$ and all $\lambda \in \mathfrak{a}_{P \mathbb{C}}^{*}$.

Proof. It clearly suffices to prove the result for $d=1$ and $D \in \mathfrak{g}$. We use the decomposition $\mathfrak{g}=\mathfrak{n}_{P} \oplus \mathfrak{a}_{P} \oplus\left(\mathfrak{m}_{P} \cap \mathfrak{p}\right) \oplus \mathfrak{k}$. Since $\mathfrak{k}$ is $\operatorname{Ad}(K)$-invariant, it follows that

$$
\mathfrak{g}=\operatorname{Ad}\left(k^{-1}\right)\left(\mathfrak{n}_{P} \oplus \mathfrak{a}_{P} \oplus\left(\mathfrak{m}_{P} \cap \mathfrak{p}\right)\right) \oplus \mathfrak{k}
$$

for each $k \in K$. Accordingly, we write

$$
D=\operatorname{Ad}\left(k^{-1}\right)\left(U_{k}+V_{k}+W_{k}\right)+Z_{k} .
$$

The elements $U_{k} \in \mathfrak{n}_{P}, V_{k} \in \mathfrak{a}_{P}, W_{k} \in \mathfrak{m}_{P} \cap \mathfrak{p}$ and $Z_{k} \in \mathfrak{k}$ all depend smoothly on $k \in K$.

If $f \in C^{\infty}(K: \xi)$ and $\lambda \in \mathfrak{a}_{P \mathbb{C}}^{*}$, then by $f_{\lambda}$ we denote the unique function in $C^{\infty}(P: \xi: \lambda)$ that restricts to $f$ on $K$. Then

$$
\begin{aligned}
& {\left[\pi_{P, \xi, \lambda}(D) f\right](k)} \\
& \quad=\left[L_{-U_{k}-V_{k}-W_{k}} f_{\lambda}\right](k)+\left[R_{Z_{k}} f\right](k) \\
& \quad=\left(\lambda+\rho_{P}\right)\left(V_{k}\right) f(k)+\xi\left(W_{k}\right)(f(k))+\left[R_{Z_{k}} f\right](k) .
\end{aligned}
$$

In order to estimate $\left\|\pi_{P, \xi, \lambda}(D) f\right\|_{s}$ we use the definition (1.9). Let $n$ be a multi-index with $|n| \leq s$, and apply $X^{n} \in \mathcal{U}(\mathfrak{k})$ to (1.12), as a function of $k$. We consider the three terms separately.

Since $k \mapsto V_{k}$ is smooth, the derivatives of $k \mapsto\left(\lambda+\rho_{P}\right)\left(V_{k}\right)$ up to order $s$ are bounded uniformly by a constant times $1+|\lambda|$. By the Leibniz rule, it then follows that

$$
\left\|R_{X^{n}}\left[k \mapsto\left(\lambda+\rho_{P}\right)\left(V_{k}\right) f(k)\right]\right\|_{0} \leq C(1+|\lambda|)\|f\|_{s}
$$


for some constant $C>0$.

Let $W \in \mathfrak{m}_{P}$. Then $\xi(W)$ maps $\mathcal{H}_{\xi}^{\infty}$ continuously into itself. By assumption (b) in the definition of $\mathcal{R}\left(M_{P}\right)$ there exists a finite subset $F \subset U\left(\mathfrak{m}_{P} \cap \mathfrak{k}\right)$ such that

$$
\|\xi(W) a\|_{\xi} \leq \max _{u \in F}\|\xi(u) a\|_{\xi}, \quad\left(a \in \mathcal{H}_{\xi}^{\infty}\right) .
$$

This in turn implies that there exist constants $r \in \mathbb{N}$ and $C>0$ such that, for all $f \in C^{\infty}(K: \xi)$ and $k \in K$,

$$
\begin{aligned}
\|\xi(W)(f(k))\|_{\xi} & \leq \max _{u \in F}\|\xi(u)(f(k))\|_{\xi} \\
& \left.=\max _{u \in F} \|\left[L_{u^{\vee}} f\right](k)\right) \|_{\xi} \\
& =\max _{u \in F}\left\|\left[R_{\operatorname{Ad}(k)^{-1} u} f\right](k)\right\|_{\xi} \\
& \leq C\|f\|_{r} .
\end{aligned}
$$

Since the right action of $K$ commutes with the application of $\xi(W)$ in this expression, we conclude that the derivatives of $k \mapsto \xi(W)(f(k))$ up to order $s$ are bounded by $C\|f\|_{r+s}$. It follows that

$$
\left\|R_{X^{n}}\left[k \mapsto \xi\left(W_{k}\right)(f(k))\right]\right\|_{0} \leq C\|f\|_{r+s},
$$

for some constant $C>0$.

Finally, it is clear that

$$
\left\|R_{X^{n}}\left[k \mapsto R_{Z_{k}} f(k)\right]\right\|_{0} \leq C\|f\|_{s+1}
$$

for some constant $C>0$. The lemma now follows with $t=\max \{r, 1\}$.

We proceed by proving an estimate from which we shall derive a more general estimate in Theorem 1.5, by product decomposition. We need the following simple observation.

Lemma 1.3 $\left\langle\rho_{P}, \alpha\right\rangle>0$ for all $\alpha \in \Sigma\left(P, \mathfrak{a}_{P}\right)$.

Proof. Let $P_{0} \subset P$ be a minimal parabolic subgroup containing $A_{0}$. Then it is well known that $\left\langle\rho_{P_{0}}, \alpha\right\rangle>0$ for all $\alpha \in \Sigma\left(P_{0}, \mathfrak{a}_{0}\right)$. The latter set is a positive system for $\Sigma\left(\mathfrak{g}, \mathfrak{a}_{0}\right)$; let $\Delta\left(P_{0}\right)$ be the subset of simple roots.

Put $\mathfrak{a}_{M_{P}}:=\mathfrak{a}_{0} \cap \mathfrak{m}_{P}$ and let $\rho_{M_{P}} \in \mathfrak{a}_{M_{P}}^{*}$ be the $\rho$ of the minimal parabolic subgroup $P_{0} \cap M_{P}$ of $M_{P}$. Then $\rho_{P_{0}}=\rho_{M_{P}}+\rho_{P}$, by [17, eq. (1.11), and this decomposition is compatible with the decomposition $\mathfrak{a}_{0}=\mathfrak{a}_{M_{P}} \oplus \mathfrak{a}_{P}$.

Let now $\alpha \in \Delta\left(P_{0}\right)$. If $\alpha$ restricts to zero on $\mathfrak{a}_{P}$, then $\left\langle\rho_{P}, \alpha\right\rangle=0$. On the other hand, if $\alpha$ does not restrict to zero on $\mathfrak{a}_{P}$, then $\left\langle\rho_{M_{P}}, \alpha\right\rangle \leq 0$ and we see that $\left\langle\rho_{P}, \alpha\right\rangle>0$ for such $\alpha$. From these properties of the simple roots it follows that for each $\alpha \in \Sigma\left(P_{0}, \mathfrak{a}_{0}\right)$ the inner product $\left\langle\rho_{P}, \alpha\right\rangle$ is positive as soon as $\left.\alpha\right|_{\mathfrak{a}_{P}} \neq 0$. The non-zero restrictions $\mathfrak{a}_{\mathfrak{a}_{P}}$ constitute $\Sigma\left(P, \mathfrak{a}_{P}\right)$, and since $\rho_{P}=0$ on $\mathfrak{a}_{M_{P}}$, the claim follows. 
Corollary 1.4 Let $P \in \mathcal{P}$ and $\xi \in \mathcal{R}\left(M_{P}\right)$. Then for every $f \in C^{\infty}(K: \xi)$ the $C^{\infty}(K: \xi)$-valued function $\lambda \mapsto A(\bar{P}: P: \xi: \lambda) f$ extends meromorphically to $\mathfrak{a}_{P \mathbb{C}}^{*}$.

For every $R \in \mathbb{R}$ there exists a polynomial function $q: \mathfrak{a}_{P \mathbb{C}}^{*} \rightarrow \mathbb{C}$ and constants $N \in \mathbb{N}$ and $r \in \mathbb{N}$ with the following properties.

(a) For every $f \in C^{\infty}(K: \xi)$ the $C^{\infty}(K: \xi)$-valued meromorphic function $\lambda \mapsto q(\lambda) A(\bar{P}: P: \xi: \lambda) f$ is regular on $\mathfrak{a}_{P}^{*}(\bar{P} \mid P, R)$.

(b) For every $s \in \mathbb{N}$ there exists a constant $C>0$ such that, for all $f \in$ $C^{\infty}(K: \xi)$ and all $\lambda \in \mathfrak{a}_{P}^{*}(\bar{P} \mid P, R)$,

$$
\|q(\lambda) A(\bar{P}: P: \xi: \lambda) f\|_{s} \leq C(1+|\lambda|)^{N}\|f\|_{s+r} .
$$

Proof. We will show that $A(\lambda):=A(\bar{P}: P: \xi: \lambda)$ extends meromorphically to $\mathfrak{a}_{P}^{*}(\bar{P} \mid P, R)$, with the required estimates, by downward induction on $R \in \mathbb{R}$. If $R \geq r_{\xi}$ then $A(\lambda)$ depends holomorphically on $\lambda \in \mathfrak{a}_{P}(\bar{P} \mid P, R)$, and (a) and (b) are valid by (1.10), with $q=1, N=1$ and $r=0$.

Let $c$ be the minimum value of $4\left\langle\alpha, \rho_{P}\right\rangle$ as $\alpha \in \Sigma\left(P, \mathfrak{a}_{P}\right)$. It follows from Lemma 1.3 that $c>0$. Let $R_{1} \in \mathbb{R}$. Assuming that the claimed result has been established for $R=R_{1}$, we will show that it is valid for $R=R_{1}-c$. Note that with this choice of $R$ the set $\mathfrak{a}_{P}^{*}(\bar{P} \mid P, R)+4 \rho_{P}$ is contained in $\mathfrak{a}_{P}^{*}\left(\bar{P} \mid P, R_{1}\right)$.

Let $q_{1}: \mathfrak{a}_{P \mathbb{C}}^{*} \rightarrow \mathbb{C}$ be a polynomial and $N_{1}, r_{1} \in \mathbb{N}$ constants, such that (a) and (b) hold for $R=R_{1}$, and let $b_{\xi}$ and $D$ be as in Theorem 1.1. also for $R=R_{1}$. Then we will verify (a) and (b) for $R=R_{1}-c$ with $q(\lambda):=b_{\xi}(\lambda) q_{1}\left(\lambda+4 \rho_{P}\right)$.

We recall that $D$ is a polynomial on $\mathfrak{a}_{P \mathbb{C}}^{*}$, with values in $U(\mathfrak{g})$. This means that we can write $D(\lambda)$ as a linear combination of finitely many fixed elements from $U(\mathfrak{g})$, say of order $\leq d$, with coefficients that depend polynomially on $\lambda$, say of degree $\leq m$.

Let $f \in C^{\infty}(K: \xi)$. Then by analytic continuation, (1.7) holds for $\lambda \in$ $\mathfrak{a}_{P}^{*}\left(\bar{P} \mid P, R_{1}\right)$. It follows that we have the following identity of meromorphic $C^{\infty}(K: \xi)$-valued functions in the variable $\lambda \in \mathfrak{a}_{P}^{*}\left(\bar{P} \mid P, R_{1}\right)$ :

$$
\begin{aligned}
q(\lambda) A(\lambda) f & =b_{\xi}(\lambda) q_{1}\left(\lambda+4 \rho_{P}\right) A(\lambda) f \\
& =q_{1}\left(\lambda+4 \rho_{P}\right) A\left(\lambda+4 \rho_{P}\right) \pi_{P, \xi, \lambda+4 \rho_{P}}(D(\lambda)) f .
\end{aligned}
$$

The last expression extends holomorphically to $\lambda+4 \rho_{P} \in \mathfrak{a}_{P}^{*}\left(\bar{P}, \mid P, R_{1}\right)$, hence in particular to $\lambda \in \mathfrak{a}_{P}^{*}(\bar{P} \mid P, R)$, and therefore so does $q(\lambda) A(\lambda) f$. The required estimate follows by application of the induction hypothesis and Lemma 1.2 ,

$$
\begin{aligned}
\|q(\lambda) A(\lambda) f\|_{s} & =\left\|q_{1}\left(\lambda+4 \rho_{P}\right) A\left(\lambda+4 \rho_{P}\right) \pi_{P, \xi, \lambda+4 \rho_{P}}(D(\lambda)) f\right\|_{s} \\
& \leq C_{1}(1+|\lambda|)^{N_{1}}\left\|\pi_{P, \xi, \lambda+4 \rho_{P}}(D(\lambda)) f\right\|_{s+r_{1}} \\
& \leq C_{2}(1+|\lambda|)^{N_{1}+m+d}\|f\|_{s+r_{1}+t} .
\end{aligned}
$$

The above result can be refined as follows. If $V$ is a finite dimensional real vector space equipped with a positive definite inner product, and $S \subset V \backslash\{0\}$, we denote by $\Pi_{S}(V)$ the set of polynomial functions on $V_{\mathbb{C}}$, which are products of first order polynomials of the form $\lambda \mapsto\langle\lambda, \alpha\rangle-c$ with $\alpha \in S$ and $c \in \mathbb{C}$. We define $\Pi_{S, \mathbb{R}}(V)$ similarly, but with $c \in \mathbb{R}$. 
Theorem 1.5 Let $P, Q \in \mathcal{P}$ be such that $\mathfrak{a}_{P}=\mathfrak{a}_{Q}$. Let $\xi \in \mathcal{R}\left(M_{P}\right)$ and $R \in \mathbb{R}$.

There exists a polynomial function $q \in \Pi_{\Sigma\left(\bar{Q}, \mathfrak{a}_{Q}\right) \cap \Sigma\left(P, \mathfrak{a}_{P}\right)}\left(\mathfrak{a}_{P}^{*}\right)$ such that for every $f \in C^{\infty}(K: \xi)$, the function

$$
\lambda \mapsto q(\lambda) A(Q: P: \xi: \lambda) f
$$

extends to a holomorphic function on $\mathfrak{a}_{P}^{*}(Q \mid P, R)$ with values in $C^{\infty}(K: \xi)$, for which the following estimates hold:

There exist $r \in \mathbb{N}, N \in \mathbb{N}$ and for every $s \in \mathbb{N}$ a constant $C>0$, such that

$$
\|q(\lambda) A(Q: P: \xi: \lambda) f\|_{s} \leq C(1+|\lambda|)^{N}\|f\|_{s+r},
$$

for all $f \in C^{\infty}(K: \xi)$ and all $\lambda \in \mathfrak{a}_{P}^{*}(Q \mid P, R)$.

Proof. We first recall that the operator $A(Q: P: \xi: \lambda)$ may be expressed as a composition of similar operators with $Q$ and $P$ adjacent, i.e., the chambers $\mathfrak{a}_{P}^{+}$and $\mathfrak{a}_{Q}^{+}$are adjacent in $\mathfrak{a}_{P}$ or, equivalently, the roots in $\Sigma\left(\bar{Q}, \mathfrak{a}_{P}\right) \cap \Sigma\left(P, \mathfrak{a}_{P}\right)$ are proportional; see [14], Sect. I.2, [17, Sect. 7, and 21] for details. The result is now readily reduced to the case that $Q$ and $P$ are adjacent. From now on, we assume this to be the case.

There exists precisely one reduced root $\alpha \in \Sigma\left(P, \mathfrak{a}_{P}\right)$ such that $\overline{\mathfrak{n}}_{Q} \cap \mathfrak{n}_{P}=$ $\mathfrak{n}_{\alpha}:=\sum_{c>0} \mathfrak{g}_{c \alpha}$. Fix an element $X \in \operatorname{ker} \alpha \cap \overline{\mathfrak{a}}_{P}^{+}$such that $\alpha$ is the unique reduced root in $\Sigma\left(P, \mathfrak{a}_{P}\right)$ vanishing on $X$. Let $\Lambda \subset \mathbb{R}$ be the collection of nonnegative weights of $\operatorname{ad} X$ in $\mathfrak{g}$. The sum $\mathfrak{s}:=\sum_{\lambda \in \Lambda} \mathfrak{g}_{\lambda}$ of the associated weight spaces is a parabolic subalgebra of $\mathfrak{g}$. Let $S=M_{S} A_{S} N_{S}$ be the associated parabolic subgroup in $G$ with the indicated Langlands decomposition. Then

$$
\mathfrak{m}_{S}=\mathfrak{m}_{P} \oplus \mathfrak{a}_{\alpha} \oplus \mathfrak{n}_{\alpha} \oplus \overline{\mathfrak{n}}_{\alpha},
$$

where $\mathfrak{a}_{\alpha}$ is the (one dimensional) orthocomplement of ker $\alpha$ in $\mathfrak{a}_{P}$. We now observe that ${ }^{*} P:=P \cap M_{S}$ and ${ }^{*} Q:=Q \cap M_{S}$ are (maximal) parabolic subgroups of $M_{S}$ with Langlands decompositions ${ }^{*} P=M_{P} A_{\alpha} N_{\alpha}$ and ${ }^{*} Q=M_{P} A_{\alpha} \bar{N}_{\alpha}$. In particular, ${ }^{*} P$ and ${ }^{*} Q$ are opposite. From the integral formulas for the standard intertwining operators, it follows that, for all $f \in C^{\infty}(K: \xi), k \in K$,

$$
[A(Q: P: \xi: \lambda) f](k)=A\left({ }^{*} Q:{ }^{*} P: \xi:\left.\lambda\right|_{\mathfrak{a}_{\alpha}}\right)\left[\left.R_{k} f\right|_{M_{S}}\right](e),
$$

for $\lambda \in \mathfrak{a}_{P \mathbb{C}}^{*}$ with $\langle\operatorname{Re} \lambda, \beta\rangle>c_{\xi}$ for all $\beta \in \Sigma\left(P, \mathfrak{a}_{P}\right)$. From this it follows by application of Corollary 1.4 that the expression on the left-hand side has a meromorphic extension as a $\mathcal{H}_{\xi}$-valued function of $\lambda \in \mathfrak{a}_{P \mathbb{C}}^{*}$. Moreover, this function depends on $\lambda$ through the one-dimensional restriction $\left.\lambda\right|_{\mathfrak{a}_{\alpha}}$. As $\left.R_{k} f\right|_{K_{S}} \in$ $C^{\infty}\left(K_{S}: \xi\right)$ depends continuously on $k$, it follows that $A(Q: P: \xi: \lambda) f$ is a meromorphic function of $\left.\lambda\right|_{\mathfrak{a}_{\alpha}}$ with values in $C(K: \xi)$.

Let now $R \in \mathbb{R}$ and let ${ }^{*} q, N$ and $r$ be associated with the data $M_{S},{ }^{*} P, \xi, R$ as in Corollary 1.4. Put $q(\lambda):={ }^{*} q\left(\left.\lambda\right|_{\mathfrak{a}_{\alpha}}\right)$. Then, clearly, $q \in \Pi_{\{\alpha\}}\left(\mathfrak{a}_{P}^{*}\right)$. Moreover, if $\lambda \in \mathfrak{a}_{P}^{*}(Q \mid P, R)$, then $\left.\lambda\right|_{\mathfrak{a}_{\alpha}} \in \mathfrak{a}_{\alpha}^{*}\left(\left.{ }^{*} Q\right|^{*} P, R\right)$. It follows that the $C(K: \xi)$ valued meromorphic function $\lambda \mapsto q(\lambda) A(Q: P: \xi: \lambda) f$ is regular on $\mathfrak{a}_{P}^{*}(Q \mid P, R)$ and satisfies the estimate

$$
\begin{aligned}
\|q(\lambda) A(Q: P: \xi: \lambda) f\|_{0} & \leq \sup _{k \in K}{ }^{*} C\left(1+\left|\left(\left.\lambda\right|_{\mathfrak{a}_{\alpha}}\right)\right| \|\right)^{N}\left\|R_{k} f\right\|_{C^{r}\left(K_{S}: \xi\right)} \\
& \leq C(1+|\lambda|)^{N}\|f\|_{r}
\end{aligned}
$$


with $C>0$ a constant independent of $f$. By analytic continuation it follows that the operator $A(Q: P: \xi: \lambda)$ intertwines the representations $\pi_{P, \xi, \lambda}$ and $\pi_{Q, \xi, \lambda}$ for generic $\lambda \in \mathfrak{a}_{P \mathbb{C}}^{*}$. In particular, it is $K$-intertwining, and the estimate (1.14) readily follows from (1.15). The proof is now easily completed.

Remark 1.6 Although we do not need it in the sequel, we note that if $\xi$ has real infinitesimal character then Thm. 1.5 can be improved so that $q \in$ $\Pi_{\Sigma\left(\bar{Q}, \mathfrak{a}_{Q}\right) \cap \Sigma\left(P, \mathfrak{a}_{P}\right), \mathbb{R}}\left(\mathfrak{a}_{P}^{*}\right)$. This can be seen from the stated version of the theorem together with [17, Theorem 6.6, by means of the argument in Remark 3.2 below.

\section{Normalized Eisenstein integrals and $c$-functions}

In the rest of this paper we keep assuming that $G$ is a group of the HarishChandra class unless specified otherwise. Let $\sigma$ be an involution of $G$ and $H$ an open subgroup of the group $G^{\sigma}$ of fixed points for $\sigma$. Then $\mathrm{X}:=G / H$ is a reductive symmetric space of the Harish-Chandra class.

There exists a Cartan involution $\theta$ which commutes with $\sigma$. The associated maximal compact subgroup is denoted by $K$. We have the decompositions $\mathfrak{g}=$ $\mathfrak{k} \oplus \mathfrak{p}=\mathfrak{h} \oplus \mathfrak{q}$ into direct sums of the +1 and -1 eigenspaces of the infinitesimal involutions $\theta$ and $\sigma$, respectively. We fix an inner product $\langle\cdot, \cdot\rangle$ on $\mathfrak{g}$ as in the beginning of Section 1, subject to the additional requirement that the involution $\sigma$ be symmetric with respect to it.

We fix a maximal abelian subspace $\mathfrak{a}_{\mathrm{q}}$ of $\mathfrak{p} \cap \mathfrak{q}$ and extend it to a maximal abelian subspace $\mathfrak{a}_{0}$ of $\mathfrak{p}$. Of course the results of the previous section are available for the present choices of $K$ and $\mathfrak{a}_{0}$.

We fix a finite dimensional unitary representation $\left(\tau, V_{\tau}\right)$ of $K$ and consider the following space of smooth $\tau$-spherical functions on $\mathrm{X}$ :

$$
C^{\infty}(\mathrm{X}: \tau):=\left\{f \in C^{\infty}(\mathrm{X}, V) \mid f(k x)=\tau(k) f(x) \forall x \in \mathrm{X}, k \in K\right\} .
$$

Let $A_{\mathrm{q}}:=\exp \mathfrak{a}_{\mathrm{q}}$. Then $G=K A_{\mathrm{q}} H$, hence a function from the space (2.1) is completely determined by its restriction to $A_{\mathrm{q}}$.

The restricted root system $\Sigma$ of $\mathfrak{a}_{\mathrm{q}}$ in $\mathfrak{g}$ is a possibly non-reduced root system. The associated collection of regular points in $A_{\mathrm{q}}$ is denoted by $A_{\mathrm{q}}^{\mathrm{reg}}$. We recall that the set $\mathrm{X}_{+}:=K A_{\mathrm{q}}^{\mathrm{reg}} H$ is open dense in $\mathrm{X}$.

Let $\mathcal{P}_{\sigma}^{\min }$ denote the set of minimal $\sigma \theta$-stable parabolic subgroups of $G$ containing $A_{\mathrm{q}}$. Then $\mathcal{P}_{\sigma}^{\min } \subset \mathcal{P}$. From [2], $\S 2$, we recall that each $P \in \mathcal{P}_{\sigma}^{\min }$ has a Langlands decomposition of the form

$$
P=M A N_{P},
$$

with $M=M_{P}$ and $A=A_{P}$ independent of $P$ and defined as follows. Let $M_{1}$ denote the centralizer of $\mathfrak{a}_{\mathrm{q}}$ in $\mathfrak{g}$, then $M_{1}=M A$ where $M={ }^{\circ} M_{1}$ (see [20], Part II, p. 18 for this notation) and $A=\exp \left(\operatorname{center}\left(\mathfrak{m}_{1}\right) \cap \mathfrak{p}\right)$. We also recall that $\mathfrak{a}_{\mathrm{q}} \subset \mathfrak{a} \subset \mathfrak{a}_{0}$. Moreover, the space $\mathfrak{a}$ is $\sigma$-invariant, and $\mathfrak{a} \cap \mathfrak{q}=\mathfrak{a}_{\mathrm{q}}$, so that

$$
\mathfrak{a}=(\mathfrak{a} \cap \mathfrak{h}) \oplus \mathfrak{a}_{\mathrm{q}} .
$$


For each $P \in \mathcal{P}_{\sigma}^{\min }$, we denote by $\Sigma(P)$ the collection of $\mathfrak{a}_{\mathrm{q}}$-weights in $\mathfrak{n}_{P}$. Then $\Sigma(P)$ is a positive system for $\Sigma$; the associated open Weyl chamber in $A_{\mathrm{q}}$ is denoted by $A_{\mathrm{q}}^{+}(P)$. By [2], Lemma 2.8, the map $Q \mapsto \Sigma(Q)$ is a bijection from $\mathcal{P}_{\sigma}^{\min }$ onto the collection of positive systems for $\Sigma$.

The image of the natural embedding $N_{K}\left(\mathfrak{a}_{\mathrm{q}}\right) / Z_{K}\left(\mathfrak{a}_{\mathrm{q}}\right) \hookrightarrow \mathrm{GL}\left(\mathfrak{a}_{\mathrm{q}}\right)$ equals the Weyl group $W$ of $\Sigma$. The image of $N_{K \cap H}\left(\mathfrak{a}_{\mathrm{q}}\right)$ in $W$ is denoted by $W_{K \cap H}$. We fix a complete set of representatives $\mathcal{W}$ of $W / W_{K \cap H}$ in $N_{K}\left(\mathfrak{a}_{\mathrm{q}}\right)$. Then for each $Q \in \mathcal{P}_{\sigma}^{\min }$ we have the disjoint union

$$
\mathrm{X}_{+}=\cup_{v \in \mathcal{W}} K A_{\mathrm{q}}^{+}(Q) v H .
$$

Consequently, any function from (2.1) is completely determined by its restrictions to $A_{\mathrm{q}}^{+}(Q) v$, for $v \in \mathcal{W}$.

We denote by $\widehat{M}_{\mathrm{fu}}$ the collection of (equivalence classes) of finite dimensional irreducible unitary representations $\xi$ of $M$, and by $\widehat{M}_{\mathrm{ps}}$ the subcollection of those $\xi$ for which there exists $v \in \mathcal{W}$ such that $\xi$ has a non-trivial $M \cap v H v^{-1}$ fixed vector. Of course these representations are trivial on the non-compact factors of $M$. If $P \in \mathcal{P}_{\sigma}^{\min }$, then the associated series of induced representations $\operatorname{Ind}_{P}^{G}(\xi \otimes \lambda \otimes 1)$, for $\xi \in \widehat{M}_{\mathrm{ps}}$ and $\lambda \in \mathfrak{a}_{\mathrm{qC}}^{*}$, is called the minimal principal series for $\mathrm{X}$, see [2], $\S 3$.

Associated with $\tau$ and this series of representations, the normalized Eisenstein integral $E^{\circ}(P: \lambda)$ is defined as in [4], $\S 5$. Let

$$
{ }^{\circ} \mathcal{C}(\tau):=\oplus_{v \in \mathcal{W}} C^{\infty}\left(M / M \cap v H v^{-1}: \tau_{M}\right),
$$

with $\tau_{M}:=\left.\tau\right|_{M \cap K}$. This space was denoted $\mathcal{A}_{2, P}$ in [7. Equipped with the direct sum of the $L^{2}$-inner products on the summands, it is a finite dimensional Hilbert space.

The Eisenstein integral is a smooth function on $\mathrm{X}$, with values in the space $\operatorname{Hom}\left({ }^{\circ} \mathcal{C}(\tau), V_{\tau}\right)$, and depending meromorphically on the parameter $\lambda \in \mathfrak{a}_{\mathrm{qC}}^{*}$. For each $\psi \in{ }^{\circ} \mathcal{C}(\tau)$, and regular $\lambda \in \mathfrak{a}_{\mathrm{qC}}^{*}$, the function $E^{\circ}(P: \lambda) \psi$ belongs to $C^{\infty}(\mathrm{X}: \tau)$ and is finite for the algebra $\mathbb{D}(\mathrm{X})$ of invariant differential operators on X. Accordingly, it has a convergent series expansion on each region $K A_{\mathrm{q}}^{+}(Q) v H$, for $Q \in \mathcal{P}_{\sigma}^{\min }$ and $v \in \mathcal{W}$. The normalized $c$-functions $C_{Q \mid P}^{\circ}(s: \lambda)$, for $s \in W$, are determined by the leading coefficients in these expansions. More precisely, each function $C_{Q \mid P}^{\circ}(s:$ : $)$ is a meromorphic function on $\mathfrak{a}_{\mathrm{qC}}^{*}$ with values in $\operatorname{End}\left({ }^{\circ} \mathcal{C}(\tau)\right)$. On $i \mathfrak{a}_{\mathrm{q}}^{*}$ it is regular and unitary, and for $\lambda \in i \mathfrak{a}_{\mathrm{q}}^{*}$ the top order asymptotic behavior of the Eisenstein integral along $K A_{\mathrm{q}}^{+}(Q) v H$ is described by the formula

$$
E^{\circ}(P: \lambda: \text { mav }) \psi \sim \sum_{s \in W} a^{s \lambda-\rho_{Q}}\left[C_{Q \mid P}^{\circ}(s: \lambda) \psi\right]_{v}(m),
$$

as $m \in M$, and $a \rightarrow \infty$ in $A_{\mathrm{q}}^{+}(Q)$. Here $v \in \mathcal{W}$ and the subscript indicates that the $v$-component of an element in the space (2.4) has been taken.

Let $P \in \mathcal{P}$ be fixed, and write $C^{\circ}(s: \lambda):=C_{P \mid P}^{\circ}(s: \lambda)$. The following result is stated without proof in [9], Thm. 10.1. We will give the proof in the remainder of this article. The set of polynomials $\Pi_{\Sigma, \mathbb{R}}\left(\mathfrak{a}_{\mathrm{q}}^{*}\right)$ is defined above Thm. 1.5. 
Theorem 2.1 Let $s \in W$ and let $\omega \subset \mathfrak{a}_{\mathrm{q}}^{*}$ be compact. There exists a polynomial $q \in \Pi_{\Sigma, \mathbb{R}}\left(\mathfrak{a}_{\mathrm{q}}^{*}\right)$ and a number $N \in \mathbb{N}$ such that $\lambda \mapsto(1+|\lambda|)^{-N} q(\lambda) C^{\circ}(s: \lambda)$ is bounded on $\omega+i \mathfrak{a}_{\mathrm{q}}^{*}$.

\section{Polynomial estimates for the $c$-functions}

In the proof of Theorem 2.1, it will be convenient to not only consider the functions $C^{\circ}(s: \lambda)$, but more generally all functions $C_{Q \mid P}^{\circ}(s: \lambda)$, for $P, Q \in$ $\mathcal{P}_{\sigma}^{\min }$ and $s \in W$. In fact, we will establish the following result.

Theorem 3.1 Let $P, Q \in \mathcal{P}_{\sigma}^{\min }, s \in W$ and let $\omega \subset \mathfrak{a}_{\mathrm{q}}^{*}$ be compact. There exists a polynomial function $q \in \Pi_{\Sigma, \mathbb{R}}\left(\mathfrak{a}_{\mathrm{q}}^{*}\right)$, a number $N \in \mathbb{N}$ and a constant $C>0$ such that

$$
\left\|q(\lambda) C_{Q \mid P}^{\circ}(s: \lambda)\right\| \leq C(1+|\lambda|)^{N},
$$

for all $\lambda \in \omega+i \mathfrak{a}_{\mathrm{q}}^{*}$.

Remark 3.2 Notice that if (3.1) holds on $\omega^{\prime}+i \mathfrak{a}_{\mathrm{q}}^{*}$ for some compact neighborhood $\omega^{\prime}$ of $\omega$ and some polynomial function $q^{\prime} \in \Pi_{\Sigma}\left(\mathfrak{a}_{\mathrm{q}}^{*}\right)$, then it will hold on $\omega+i \mathfrak{a}_{\mathrm{q}}^{*}$ for every polynomial function $q \in \Pi_{\Sigma}\left(\mathfrak{a}_{\mathrm{q}}^{*}\right)$ for which $q(\lambda) C_{Q \mid P}^{\circ}(s: \lambda)$ is holomorphic on a neighborhood of $\omega+i \mathfrak{a}_{\mathrm{q}}^{*}$ (with constants $N$ and $C$ depending on $q$ ). This can be seen by an application of the Cauchy integral formula as in 3], proof of Lemma 6.1.

Remark 3.3 Notice also that by [4], eq. (72), the inverse of the normalized c-function is given by

$$
C_{Q \mid P}^{\circ}(s: \lambda)^{-1}=C_{P \mid Q}^{\circ}\left(s^{-1}: s \lambda\right) .
$$

Hence it follows from the estimate (3.1) that this inverse satisfies a similar bound on $\omega+i \mathfrak{a}_{\mathrm{q}}^{*}$, although of course with possibly different $q, N, C$,

$$
\left\|q(\lambda) C_{Q \mid P}^{\circ}(s: \lambda)^{-1}\right\| \leq C(1+|\lambda|)^{N} .
$$

Before we give the proof of Thm. 3.1, we will review the relation of the cfunctions to standard intertwining operators, meanwhile fixing useful notation. We recall from the previous section that every parabolic subgroup $P \in \mathcal{P}_{\sigma}^{\min }$ has a Langlands decomposition of the form (2.2), and that $\widehat{M}_{\mathrm{fu}}$ denotes the set of equivalence classes of finite dimensional irreducible unitary representations of $M$.

If $\xi \in \widehat{M}_{\mathrm{fu}}$, let $\mathcal{H}_{\xi}$ be a Hilbert space in which $\xi$ is unitarily realized. It is clear that $\widehat{M}_{\mathrm{fu}} \subset \mathcal{R}(M)$. We identify $\mathfrak{a}_{\mathrm{qC}}^{*}$ with the subspace of $\mathfrak{a}_{\mathbb{C}}^{*}$ consisting of elements vanishing on $\mathfrak{a} \cap \mathfrak{h}$. We use the notation from Section 1 for the principal series representations $\pi_{P, \xi, \lambda}$, where $\xi \in \widehat{M}_{\mathrm{fu}}$ and $\lambda \in \mathfrak{a}_{\mathrm{qC}}^{*}$. Note that $\rho_{P}=\frac{1}{2} \operatorname{tr}\left(\left.\operatorname{ad}(\cdot)\right|_{\mathfrak{n}_{P}}\right)$ is defined as a linear functional on $\mathfrak{a}$, and that it vanishes on $\mathfrak{a} \cap \mathfrak{h}$, hence belongs to $\mathfrak{a}_{\mathrm{q}}^{*}$; see [2], Lemma 3.1.

Let $d k$ be normalized Haar measure on $K$. The sesquilinear pairing

$$
C^{\infty}(P: \xi: \lambda) \times C^{\infty}(P: \xi:-\bar{\lambda}) \rightarrow \mathbb{C}
$$


given by

$$
\langle f, g\rangle=\int_{K}\langle f(k), g(k)\rangle_{\xi} d k
$$

is $G$-equivariant. In the compact picture this pairing becomes a pre-Hilbert structure on $C^{\infty}(K: \xi)$ for which $\pi_{P, \xi,-\bar{\lambda}}(x)^{*}=\pi_{P, \xi, \lambda}(x)^{-1}$, for all $x \in G$. In particular, the representation $\pi_{P, \xi, \lambda}$ is unitarizable for $\lambda \in i \mathfrak{a}_{\mathrm{q}}^{*}$.

Let $C^{-\infty}(K: \xi)$ denote the space of generalized functions $K \rightarrow \mathcal{H}_{\xi}$, transforming according to the rule (1.2), and for each $\lambda \in \mathfrak{a}_{\mathrm{qC}}^{*}$ let $C^{-\infty}(P: \xi: \lambda)$ denote the space of generalized functions $G \rightarrow \mathcal{H}_{\xi}$ transforming according to the rule (1.1). These spaces are equipped with the usual locally convex strong dual topologies of distribution spaces. By (3.4) we can identify $C^{-\infty}(K: \xi)$ with the strong anti-linear dual of the Fréchet space $C^{\infty}(K: \xi)$, and likewise we can identify $C^{-\infty}(P: \xi: \lambda)$ with the strong anti-linear dual of $C^{\infty}(P: \xi:-\bar{\lambda})$. It follows by transposition that the restriction map $\left.f \mapsto f\right|_{K}$ extends to a topological linear isomorphism from $C^{-\infty}(P: \xi: \lambda)$ onto $C^{-\infty}(K: \xi)$. The representation $\pi_{P, \xi, \lambda}$ naturally extends to a continuous representation $\pi_{P, \xi, \lambda}$ of $G$ in both of these two model spaces. The pairing (3.4) is $G$-equivariant with respect to these actions.

For $0<s<\infty$ let $C^{-s}(K: \xi)$ denote the subspace of $C^{-\infty}(K: \xi)$ consisting of the generalized functions of order at most $s$. The pairing (3.4) extends to a $K$-equivariant non-degenerate sesquilinear pairing

$$
C^{-s}(K: \xi) \times C^{s}(K: \xi) \rightarrow \mathbb{C} .
$$

Recall that we have equipped $C^{s}(K: \xi)$ with the norm $\|\cdot\|_{s}$. We equip $C^{-s}(K: \xi)$ with the dual norm, denoted $\|\cdot\|_{-s}$. Then

$$
|\langle f, g\rangle| \leq\|f\|_{-s}\|g\|_{s}
$$

for $f \in C^{-s}(K: \xi)$ and $g \in C^{s}(K: \xi)$. In this fashion $C^{-s}(K: \xi)$ becomes identified with the anti-linear dual of the Banach space $C^{s}(K: \xi)$, as a representation space for $K$.

Let $P, Q \in \mathcal{P}_{\sigma}^{\min }$. For $R \in \mathbb{R}$ we define the set

$$
\mathfrak{a}_{\mathrm{q}}^{*}(Q \mid P, R):=\left\{\lambda \in \mathfrak{a}_{\mathrm{qC}}^{*} \mid\langle\operatorname{Re} \lambda, \alpha\rangle>R, \forall \alpha \in \Sigma(\bar{Q}) \cap \Sigma(P)\right\},
$$

in analogy with (1.3).

\section{Lemma 3.4}

(a) $\Sigma(P)=\left\{\left.\alpha\right|_{\mathfrak{a}_{\mathfrak{q}}} \mid \alpha \in \Sigma(P, \mathfrak{a})\right\}$,

(b) $\Pi_{\Sigma(\bar{Q}) \cap \Sigma(P)}\left(\mathfrak{a}_{\mathrm{q}}^{*}\right)=\left\{\left.p\right|_{\mathfrak{a}_{\mathrm{qC}}^{*}} \mid p \in \Pi_{\Sigma(\bar{Q}, \mathfrak{a}) \cap \Sigma(P, \mathfrak{a})}\left(\mathfrak{a}^{*}\right)\right\}$,

(c) for every $R \in \mathbb{R}, \mathfrak{a}_{\mathrm{q}}^{*}(Q \mid P, R)=\mathfrak{a}^{*}(Q \mid P, R) \cap \mathfrak{a}_{\mathrm{q}}^{*}$.

Proof. The nilpotent radical $\mathfrak{n}_{P}$ decomposes as the direct sum of the weight spaces for $\mathfrak{a}=\mathfrak{a}_{P}$, with $\Sigma(P, \mathfrak{a})$ as the associated set of weights. The set of the right-hand side of the equation in (a) equals the set of $\mathfrak{a}_{\mathrm{q}}$-weights in $\mathfrak{n}_{P}$; this is $\Sigma(P)$. Assertion (a) follows. 
We agreed to identify $\mathfrak{a}_{\mathrm{q}}^{*}$ with the space of linear functionals in $\mathfrak{a}^{*}$ vanishing on $\mathfrak{a}_{P} \cap \mathfrak{h}$. By symmetry of $\sigma$, the decomposition (2.3) is orthogonal. This implies that the dual inner product on $\mathfrak{a}_{\mathrm{q}}^{*}$ coincides with the restriction of the dual inner product on $\mathfrak{a}^{*}$. Moreover, the restriction map $\left.\eta \mapsto \eta\right|_{\mathfrak{a}_{\mathrm{q}}}$ coincides with the orthogonal projection $\mathfrak{a}^{*} \rightarrow \mathfrak{a}_{\mathrm{q}}^{*}$. Assertions (b) and (c) now follow from (a).

It follows from Lemma 3.4 (c) that for $\lambda \in \mathfrak{a}_{\mathrm{q}}^{*}\left(Q \mid P, r_{\xi}\right)$ the intertwining operator $A(Q: P: \xi: \lambda)$ is well defined by the convergent integral (1.4). In view of Lemma 3.4 (b), (c), the following result is now an immediate consequence of Theorem 1.5.

Proposition 3.5 Let $P, Q \in \mathcal{P}_{\sigma}^{\min }$.

For every $f \in C^{\infty}(K: \xi)$ the function $\lambda \mapsto A(Q: P: \xi: \lambda) f$, originally defined on $\mathfrak{a}_{\mathrm{q}}^{*}\left(Q \mid P, r_{\xi}\right)$, extends to a meromorphic $C^{\infty}(K: \xi)$-valued function on $\mathfrak{a}_{\mathrm{qC}}^{*}$.

Let $R \in \mathbb{R}$. There exist a polynomial function $q \in \Pi_{\Sigma(\bar{Q}) \cap \Sigma(P)}\left(\mathfrak{a}_{\mathrm{q}}^{*}\right)$ and constants $r \in \mathbb{N}$ and $N \in \mathbb{N}$, such that the following is valid.

The map $\lambda \mapsto q(\lambda) A(Q: P: \xi: \lambda) f$ is holomorphic as a $C^{\infty}(K: \xi)$ valued function on $\mathfrak{a}_{\mathrm{q}}^{*}(Q \mid P, R)$, for every $f \in C^{\infty}(K: \xi)$. Moreover, for each $s \in \mathbb{N}$ there exists a constant $C>0$ such that

$$
\|q(\lambda) A(Q: P: \xi: \lambda) f\|_{s} \leq C(1+|\lambda|)^{N}\|f\|_{s+r},
$$

for all $\lambda \in \mathfrak{a}_{\mathrm{q}}^{*}(Q \mid P, R)$ and $f \in C^{\infty}(K: \xi)$.

Remark 3.6 It follows by the above estimate that, for $\lambda \in \mathfrak{a}_{\mathrm{q}}^{*}(Q \mid P, R)$, the map $A_{0}(\lambda):=q(\lambda) A(Q: P: \xi: \lambda): C^{\infty}(K: \xi) \rightarrow C(K: \xi)$ extends to a bounded linear map from $C^{s+r}(K: \xi)$ to $C^{s}(K: \xi)$, for every $s \in \mathbb{N}$.

Moreover, by a standard application of the Cauchy estimates for power series coefficients, we infer, with $R, r$ and $q$ as above, that $\lambda \mapsto A_{0}(\lambda)$ is holomorphic on $\mathfrak{a}_{\mathrm{q}}^{*}(Q \mid P, R)$ as a function with values in the Banach space $B_{s, s+r}$ of bounded linear maps $C^{s+r}(K: \xi) \rightarrow C^{s}(K: \xi)$.

With respect to the pairing (3.4) it is known that

$$
A(Q: P: \xi: \lambda)=A(P: Q: \xi:-\bar{\lambda})^{*},
$$

for generic $\lambda \in \mathfrak{a}_{\mathrm{qC}}^{*}$. In fact, this follows from the validity of this identity on the space $C^{\infty}(K: \xi)_{K}$ of $K$-finite functions in $C^{\infty}(K: \xi)$; see [17, Prop. 7.1 (iv).

It follows that the intertwining operator (1.5) admits a continuous linear extension to an intertwining operator

$$
A(Q: P: \xi: \lambda): C^{-\infty}(P: \xi: \lambda) \longrightarrow C^{-\infty}(Q: \xi: \lambda),
$$

given by the formula (3.8), for generic $\lambda \in \mathfrak{a}_{\mathrm{qC}}^{*}$.

The space of generalized functions $f \in C^{-\infty}(P: \xi: \lambda)$ which are invariant under $\pi_{P, \xi \lambda}(H)$ is denoted by $C^{-\infty}(P: \xi: \lambda)^{H}$. We need the following description of this space from [2]. 
Let $\xi \in \widehat{M}_{\mathrm{ps}}$. For $v \in \mathcal{W}$ we define $V(\xi, v)$ to be the space $\mathcal{H}_{\xi}^{M \cap v H v^{-1}}$, equipped with the restricted Hilbert inner product. Moreover, we define the space $V(\xi)$ to be the formal orthogonal direct sum

$$
V(\xi):=\oplus_{v \in \mathcal{W}} V(\xi, v) .
$$

Note that $V(\xi)$ is non-trivial by the definition of $\widehat{M}_{\mathrm{ps}}$, see Section 2 ,

From [2], Sect. 5, we recall the definition of the evaluation map

$$
\text { ev }: C^{-\infty}(P: \xi: \lambda)^{H} \rightarrow V(\xi)
$$

by

$$
[\operatorname{ev}(\varphi)]_{v}=\varphi(v), \quad(v \in \mathcal{W}) .
$$

This map is well defined, as an $H$-fixed element of $C^{-\infty}(P: \xi: \lambda)$ is smooth on each open orbit $P v H$, for $v \in \mathcal{W}$. According to [2, Thm. 5.10, there exists a unique meromorphic $\operatorname{Hom}\left(V(\xi), C^{-\infty}(K: \xi)\right)$-valued function $j(P: \xi: \cdot)$ on $\mathfrak{a}_{\mathrm{qC}}^{*}$, such that for every $\eta \in V(\xi)$ and generic $\lambda \in \mathfrak{a}_{\mathrm{qC}}^{*}$,

(a) the generalized function $j(P: \xi: \lambda) \eta$ belongs to $C^{-\infty}(P: \xi: \lambda)^{H}$;

(b) $\operatorname{ev} \circ j(P: \xi: \lambda) \eta=\eta$.

We also need the operator $B(Q: P: \xi: \lambda) \in \operatorname{End}(V(\xi))$ constructed in [2], Sect. 6. Recall that this depends meromorphically on $\lambda \in \mathfrak{a}_{\mathrm{qC}}^{*}$, and that it is uniquely determined by the relation

$$
B(Q: P: \xi: \lambda)=\operatorname{ev} \circ A(Q: P: \xi: \lambda) \circ j(P: \xi: \lambda),
$$

for generic $\lambda \in \mathfrak{a}_{\mathrm{qC}}^{*}$.

The space ${ }^{\circ} \mathrm{C}={ }^{\circ} \mathcal{C}(\tau)$ admits a direct sum decomposition of the form

$$
{ }^{\circ} \mathcal{C}(\tau)=\oplus_{\xi \in \widehat{M}_{\mathrm{ps}}}{ }^{\circ} \mathcal{C}_{\xi}(\tau),
$$

as in [4, proof of Lemma 3. All but finitely many summands of this direct sum are trivial.

Let

$$
C(K: \xi: \tau):=\left[C(K: \xi) \otimes V_{\tau}\right]^{K} .
$$

Then according to [4], Lemma 3, there exists, for each $\xi \in \widehat{M}_{\mathrm{ps}}$, a natural linear isomorphism $T \mapsto \psi_{T}$ from $C(K: \xi: \tau) \otimes \overline{V(\xi)}$ onto ${ }^{\circ} \mathcal{C}(\tau)_{\xi}$, where $\overline{V(\xi)}$ is the conjugate Hilbert space of $V(\xi)$.

Via these isomorphisms, the $c$-function $C_{Q \mid P}^{\circ}(1: \lambda)$ may be expressed in terms of standard intertwining operators, as follows. For $f \otimes \eta \in C(K: \xi$ : $\tau) \otimes \overline{V(\xi)}$, we have

$$
C_{Q \mid P}^{\circ}(1: \lambda) \psi_{f \otimes \eta}=\psi_{A(Q: P: \xi:-\lambda) f \otimes B(\bar{P}: \bar{Q}: \xi: \bar{\lambda})^{-1} \eta},
$$

as an identity of meromorphic functions in the variable $\lambda \in \mathfrak{a}_{\mathrm{qC}}^{*}$; see [4], Eqn. (57). In particular, it follows that $C_{Q \mid P}^{\circ}(1: \lambda)$ preserves the decomposition 
(3.10). It is convenient to rewrite (3.11) in the following form, which follows by application of (3.2)

$$
C_{Q \mid P}^{\circ}(1: \lambda) \psi_{f \otimes \eta}=\psi_{A(P: Q: \xi:-\lambda)^{-1} f \otimes B(\bar{Q}: \bar{P}: \xi: \bar{\lambda}) \eta} .
$$

Here we note that $\lambda \mapsto B(\bar{Q}: \bar{P}: \xi: \bar{\lambda}) \eta$ is a meromorphic $\overline{V(\xi)}$-valued function.

We now come to the proof of the theorem.

Proof of Theorem [3.1. It follows from the relation [4], Eqn. (68), that we may assume that $s=1$. Thus, we aim to prove:

$$
\left\|q(\lambda) C_{Q \mid P}^{\circ}(1: \lambda)\right\| \leq C(1+|\lambda|)^{N}, \quad\left(\lambda \in \omega+i \mathfrak{a}_{\mathrm{q}}^{*}\right) .
$$

Furthermore, it follows from (3.11) combined with the argument below Lemma 3.2 in [6], that for every compact subset $\omega^{\prime} \subset \mathfrak{a}_{\mathrm{q}}^{*}$ there exists a function $q^{\prime} \in \Pi_{\Sigma, \mathbb{R}}\left(\mathfrak{a}_{\mathrm{q}}^{*}\right)$ such that $q^{\prime}(\lambda) C_{Q \mid P}^{\circ}(1: \lambda)$ is holomorphic on a neighborhood of $\omega^{\prime}+i \mathfrak{a}_{\mathrm{q}}^{*}$. By combining this observation with that of Remark 3.2, we see that it suffices to establish (3.13) with some $q \in \Pi_{\Sigma}\left(\mathfrak{a}_{\mathrm{q}}^{*}\right)$.

From (3.10) and (3.11), and the text between and after these displays, it follows that it suffices to establish, for every $\xi \in \widehat{M}_{\mathrm{ps}}$, the existence of $q, N$ and $C$ such that the estimate (3.13) holds with $C_{Q \mid P}^{\circ}(1: \lambda)$ replaced by its restriction $C_{Q \mid P}^{\circ}(1: \lambda)_{\xi}$ to ${ }^{\circ} \mathcal{C}_{\xi}(\tau)$. We fix $\xi \in \widehat{M}_{\mathrm{ps}}$.

It follows from [3], Lemma 16.6, that for every $R \in \mathbb{R}$ there exist a polynomial $q \in \Pi_{\Sigma}\left(\mathfrak{a}_{\mathrm{q}}^{*}\right)$ and constants $N, C$ such that

$$
\left\|q(\lambda) A(P: Q: \xi:-\lambda)^{-1} f\right\|_{0} \leq C(1+|\lambda|)^{N}\|f\|_{0}
$$

for all $\lambda \in \mathfrak{a}_{\mathrm{q}}^{*}(Q \mid P, R)$ and $f \in C(K: \xi: \tau)$. In particular, this can be achieved for $\lambda \in \omega+i \mathfrak{a}_{\mathrm{q}}^{*}$ by an appropriate choice of $R$. It thus only remains to obtain a similar bound for $B(\bar{Q}: \bar{P}: \xi: \bar{\lambda}) \eta$. This is accomplished in Proposition 4.1 of the next section.

\section{Estimates for $B(Q: P: \xi: \lambda)$}

In the following it will be convenient to use the following notation for certain subsets of $\mathfrak{a}_{\mathrm{qC}}^{*}$, for given $P, Q \in \mathcal{P}_{\sigma}^{\min }$ and $R \in \mathbb{R}$ :

$$
\mathcal{A}(Q, P, R):=\left\{\lambda \in \mathfrak{a}_{\mathrm{qC}}^{*}|\forall \alpha \in \Sigma(\bar{Q}) \cap \Sigma(P):|\langle\operatorname{Re} \lambda, \alpha\rangle \mid<R\right\} .
$$

We also define

$$
\mathfrak{a}_{\mathrm{q}}^{*}(P, R):=\left\{\lambda \in \mathfrak{a}_{\mathrm{qC}}^{*} \mid\langle\operatorname{Re} \lambda, \alpha\rangle<R, \forall \alpha \in \Sigma(P)\right\},
$$

and observe that in particular

$$
\mathcal{A}(\bar{P}, P, R)=\mathfrak{a}_{\mathrm{q}}^{*}(P, R) \cap \mathfrak{a}_{\mathrm{q}}^{*}(\bar{P}, R) .
$$

Finally, we note that for each compact set $\omega \subset \mathfrak{a}_{\mathrm{q}}^{*}$ there exists $R>0$ such that $\omega+i \mathfrak{a}_{\mathrm{q}}^{*} \subset \mathcal{A}(Q, P, R)$. 
Proposition 4.1 Let $P, Q \in \mathcal{P}_{\sigma}^{\min }, \xi \in \widehat{M}_{\mathrm{ps}}$ and $R>0$. There exists a polynomial function $q \in \Pi_{\Sigma}\left(\mathfrak{a}_{\mathrm{q}}^{*}\right)$ and constants $N \in \mathbb{N}$ and $C>0$ such that the following is valid. The map $\lambda \mapsto q(\lambda) B(Q: P: \xi: \lambda)$ is a holomorphic $\operatorname{End}(V(\xi))$-valued function on the set $\mathcal{A}(Q, P, R)$. Moreover, for all $\lambda$ in this set,

$$
\|q(\lambda) B(Q: P: \xi: \lambda)\| \leq C(1+|\lambda|)^{N} .
$$

Proof. By the argument in [5], Lemma 20.5, we may assume that $Q=\bar{P}$. The condition on $\lambda$ is then that $\lambda \in \mathfrak{a}_{\mathrm{q}}^{*}(P, R) \cap \mathfrak{a}_{\mathrm{q}}^{*}(\bar{P}, R)$.

For generic $\lambda$ the endomorphism $B(\bar{P}: P: \xi: \lambda)$ of $V(\xi)$ is given as the composition of three maps in (3.9). In terms of the compact picture we will view these as maps

$$
\begin{aligned}
& j(P: \xi: \lambda): V(\xi) \rightarrow C^{-\infty}(K: \xi), \\
& A(\bar{P}: P: \xi: \lambda): C^{-\infty}(K: \xi) \rightarrow C^{-\infty}(K: \xi), \\
& \text { ev }: C^{-\infty}(K: \xi)_{\bar{P}, \lambda}^{H} \rightarrow V(\xi) .
\end{aligned}
$$

The indices $\bar{P}, \lambda$ on the domain of ev indicate that the space of $H$-invariants for the representation $\pi_{\bar{P}, \xi, \lambda}$ has been taken.

It will be seen in the following Lemmas 4.2, 4.3 and 4.4 that each of the three maps above satisfies a bound of the desired polynomial type in $\lambda$, in terms of suitable operator norms. The estimates will turn out to be valid on sets of the form $\mathfrak{a}_{\mathrm{q}}^{*}\left(P, R_{1}\right), \mathfrak{a}_{\mathrm{q}}^{*}\left(\bar{P} \mid P, R_{2}\right)$ and $\mathfrak{a}_{\mathrm{q}}^{*}\left(P, R_{3}\right)$, respectively, with $R_{j}$ arbitrary real numbers. Noting that $\mathfrak{a}_{\mathrm{q}}^{*}\left(\bar{P} \mid P, R_{2}\right)=\mathfrak{a}_{\mathrm{q}}^{*}\left(\bar{P},-R_{2}\right)$ and taking $R_{1}=R_{3}=R$ and $R_{2}=-R$ we obtain sets whose intersection equals $\mathfrak{a}_{\mathrm{q}}^{*}(P, R) \cap \mathfrak{a}_{\mathrm{q}}^{*}(\bar{P}, R)$. Thus, combining the mentioned lemmas, the proof of Proposition 4.1 is completed.

Lemma 4.2 Let $P \in \mathcal{P}_{\sigma}^{\min }, \xi \in \widehat{M}_{\mathrm{ps}}$ and $R \in \mathbb{R}$. There exist $s \in \mathbb{N}^{+}$and $q \in \Pi_{\Sigma}\left(\mathfrak{a}_{\mathrm{q}}^{*}\right)$ such that the restriction to $K$ of $q(\lambda) j(P: \xi: \lambda) \eta$ belongs to $C^{-s}(K: \xi)$ for all $\eta \in V(\xi)$ and $\lambda \in \mathfrak{a}_{\mathrm{q}}^{*}(P, R)$, and such that the norm of the restriction satisfies the following uniform estimate. There exist constants $N \in \mathbb{N}, C>0$ such that

$$
\|q(\lambda) j(P: \xi: \lambda) \eta\|_{-s} \leq C(1+|\lambda|)^{N}\|\eta\|
$$

for all $\eta \in V(\xi), \lambda \in \mathfrak{a}_{\mathrm{q}}^{*}(P, R)$.

Proof. See [3], Thm. 9.1.

We now turn to estimating a suitable operator norm of the intertwining operator (4.2). As this map is given as an adjoint in (3.8), the following lemma is the key step. In what follows, we shall use, for $p, q \in \mathbb{Z}$, the following abbreviation for the space of bounded linear maps between Banach spaces:

$$
B_{q, p}:=B\left(C^{p}(K: \xi), C^{q}(K: \xi)\right) .
$$

Moreover, we shall use the notation $\|\cdot\|_{q, p}$ for the operator norm on this space. Accordingly, if $T \in B_{q, p}$ and $T^{\prime} \in B_{r, q}$, then $T^{\prime} \circ T \in B_{r, p}$ and

$$
\left\|T^{\prime} \circ T\right\|_{r, p} \leq\left\|T^{\prime}\right\|_{r, q}\|T\|_{q, p} .
$$


Lemma 4.3 Let $P \in \mathcal{P}_{\sigma}^{\min }, \xi \in \widehat{M}_{\mathrm{fu}}$ and $R \in \mathbb{R}$. There exist a polynomial function $q \in \Pi_{\Sigma}\left(\mathfrak{a}_{\mathrm{q}}^{*}\right)$, and constants $r \in \mathbb{N}$ and $N \in \mathbb{N}$ such that the following holds.

For every $s \in \mathbb{N} \backslash\{0\}$ there exists a constant $C>0$ such that for every $g \in C^{-s}(K: \xi)$, the map $\lambda \mapsto q(\lambda) A(\bar{P}: P: \xi: \lambda) g$ is holomorphic as a $C^{-r-s}(K: \xi)$-valued function on $\mathfrak{a}_{\mathrm{q}}^{*}(\bar{P} \mid P, R)$ and

$$
\|q(\lambda) A(\bar{P}: P: \xi: \lambda) g\|_{-r-s} \leq C(1+|\lambda|)^{N}\|g\|_{-s},
$$

for all $\lambda \in \mathfrak{a}_{\mathrm{q}}^{*}(\bar{P} \mid P, R)$.

Proof. We will derive the result from Proposition 3.5 by using (3.8).

For $q \in \Pi_{\Sigma}\left(\mathfrak{a}_{\mathrm{q}}^{*}\right)$, we put $q^{\vee}(\lambda):=\overline{q(-\bar{\lambda})}$. Then $q \mapsto q^{\vee}$ is a bijection of $\Pi_{\Sigma}\left(\mathfrak{a}_{\mathrm{q}}^{*}\right)$ onto itself.

Let $s, t$ be positive integers, and let $T \in B_{t, s}$. Then we define the conjugate map $T^{*}: C^{-t}(K: \xi) \rightarrow C^{-s}(K: \xi)$ by $\left\langle T^{*} \varphi, g\right\rangle=\langle\varphi, T g\rangle$, for all $\varphi \in$ $C^{-t}(K: \xi)$ and $g \in C^{s}(K: \xi)$. It is readily seen that $T \mapsto T^{*}$ defines an anti-linear map $B_{t, s} \rightarrow B_{-s,-t}$, with

$$
\left\|T^{*}\right\|_{-s,-t} \leq\|T\|_{t, s}
$$

for all $T \in B_{t, s}$.

Let now $R \in \mathbb{R}$. Let $q_{0} \in \Pi_{\Sigma}\left(\mathfrak{a}_{\mathrm{q}}^{*}\right), r \in \mathbb{N}$ and $N \in \mathbb{N}$ be as in Proposition 3.5 with $(P, \bar{P})$ in place of $(Q, P)$. Let $s \in \mathbb{N}$ and let $C>0$ be a constant as in (3.7). According to Remark 3.6 it follows from the mentioned proposition that the map $T: \lambda \mapsto q_{0}(\lambda) A(P: \bar{P}: \xi: \lambda)$ is a holomorphic function on $\mathfrak{a}_{\mathrm{q}}^{*}(P \mid \bar{P}, R)$ with values in $B_{s, s+r}$, satisfying $\|T(\lambda)\|_{s, s+r} \leq C(1+|\lambda|)^{N}$ for all $\lambda \in \mathfrak{a}_{\mathrm{q}}^{*}(P \mid \bar{P}, R)$. This implies that, for every $s \in \mathbb{N}$, the map $\lambda \mapsto T(-\bar{\lambda})^{*}$ is a holomorphic function with values in $B_{-s-r,-s}$, satisfying the estimate

$$
\left\|T(-\bar{\lambda})^{*}\right\|_{-s-r,-s} \leq\|T(-\bar{\lambda})\|_{s, s+r} \leq C(1+|\lambda|)^{N},
$$

for all $\lambda \in-\mathfrak{a}_{\mathrm{q}}^{*}(P \mid \bar{P}, R)$. We now observe that $-\mathfrak{a}_{\mathrm{q}}^{*}(P \mid \bar{P}, R)=\mathfrak{a}_{\mathrm{q}}^{*}(\bar{P} \mid P, R)$, and that $T(-\bar{\lambda})^{*}=q_{0}^{\vee}(\lambda) A(\bar{P}: P: \xi: \lambda)$ in view of (3.8). The estimate (4.4) thus follows with $q=q_{0}^{\vee}$.

Lemma 4.4 Let $P \in \mathcal{P}_{\sigma}^{\min }, \xi \in \widehat{M}_{\mathrm{ps}}, R \in \mathbb{R}$ and $s \in \mathbb{N}$. There exists a constant $C>0$ such that

$$
\| \text { ev } g\left\|\leq C(1+|\lambda|)^{s}\right\| g \|_{-s}
$$

for all $\lambda \in \mathfrak{a}_{\mathrm{q}}^{*}(\bar{P}, R)$ and $g \in C^{-s}(K: \xi) \cap C^{-\infty}(K: \xi)_{P, \lambda}^{H}$.

The proof of this lemma will be given in the next section. 


\section{Estimation of the evaluation map}

This section is entirely devoted to the proof of Lemma 4.4. To prepare for it, we first make the following general observation.

Let $G$ be a Lie group, and let $P$ be a closed subgroup. Then the adjoint action of $P$ on $\mathfrak{g}$ naturally induces a left action of $P$ on the space of densities on $\mathfrak{g} / \mathfrak{p}$. As the latter space is one-dimensional, the action is given by a character $\chi: P \rightarrow \mathbb{R}^{+}$. In fact, this character is given by

$$
\chi(p)=\frac{\left|\operatorname{det} \operatorname{Ad}_{P}(p)\right|}{\left|\operatorname{det} \operatorname{Ad}_{G}(p)\right|}, \quad(p \in P) .
$$

We consider the space $C(G: P: \chi)$ of continuous functions $f: G \rightarrow \mathbb{C}$ such that $f(p g)=\chi(p) f(g)$ for all $g \in G$ and $p \in P$ and write $C_{c}(G: P: \chi)$ for the subspace of functions with compact support modulo $P$.

Let $\mathcal{D}_{P \backslash G}$ denote the density bundle on $P \backslash G$. Its fiber at a point $P g$ is the space $\mathcal{D}\left(T_{P g}(P \backslash G)\right)$ of densities on the tangent space $T_{P g}(P \backslash G)$. Pull-back by right multiplication gives a linear isomorphism $d\left(r_{g^{-1}}\right)(P g)^{*}: \mathcal{D}\left(T_{P e}(P \backslash G)\right) \rightarrow$ $\mathcal{D}\left(T_{P g}(P \backslash G)\right)$.

Let $\Gamma_{c}\left(\mathcal{D}_{P \backslash G}\right)$ denote the space of compactly supported continuous sections of $\mathcal{D}(P \backslash G)$. We fix a positive density $\omega$ on $T_{P e}(P \backslash G) \simeq \mathfrak{g} / \mathfrak{p}$. Then the map $f \mapsto j_{\omega}(f)$ given by

$$
j_{\omega}(f)(P g)=f(g) d\left(r_{g^{-1}}\right)(P g)^{*} \omega
$$

defines a topological linear isomorphism

$$
j_{\omega}: C_{c}(G: P: \chi) \stackrel{\simeq}{\longrightarrow} \Gamma_{c}\left(\mathcal{D}_{P \backslash G}\right) .
$$

Lemma 5.1 Let $L$ be a closed subgroup of $G$ such that $P L$ is open in $G$ and such that $\left.\chi\right|_{L \cap P}=1$. Then there exists a unique right-invariant positive Radon measure $d \bar{l}$ on $(L \cap P) \backslash L$ such that

$$
\int_{P \backslash G} j_{\omega}(f)=\int_{(L \cap P) \backslash L} f(l) d \bar{l}
$$

for all $f \in C_{c}(G: P: \chi)$ with $\operatorname{supp} f \subset P L$.

Proof. Let $C_{L}(G: P: \chi)$ denote the space of functions $f$ as in the lemma. Then $\left.f \mapsto f\right|_{L}$ defines a topological linear isomorphism from $C_{L}(G: P: \chi)$ onto $C_{c}((L \cap P) \backslash L)$. We denote its inverse by $g \mapsto \tilde{g}$. The functional $\mu: g \mapsto$ $\int_{P \backslash G} j_{\omega}(\tilde{g})$ defines a Radon measure on $(P \cap L) \backslash L$. Let $m \in L$. As the integration of densities is invariant under pull-back by diffeomorphisms, we find, for $g \in$ $C_{c}((L \cap P) \backslash L)$, that

$$
\mu\left(r_{m}^{*} g\right)=\int_{P \backslash G} j_{\omega}\left(\widetilde{r_{m}^{*} g}\right)=\int_{P \backslash G} r_{m}^{*}\left(j_{\omega}(\tilde{g})\right)=\int_{P \backslash G} j_{\omega}(\tilde{g})=\mu(g) .
$$

Hence, $\mu$ is right $L$-invariant and the result follows with $d \bar{l}=\mu$. Uniqueness is obvious. 
We now return to the notation of the previous sections, with $G$ a group of the Harish-Chandra class, and apply Lemma 5.1 with $P$ a parabolic subgroup from $\mathcal{P}_{\sigma}^{\min }$. Then

$$
\chi(\operatorname{man})=a^{2 \rho_{P}}, \quad\left(m \in M_{P}, a \in A_{P}, n \in N_{P}\right) .
$$

It follows that $\chi=1$ on both $K \cap P=K \cap M_{P}$ and $H \cap P=\left(H \cap M_{P}\right) A_{H}$. So we may apply Lemma 5.1 with $L$ equal to $K$ and with $L$ equal to $H$.

It follows that we may fix the density $\omega$ on $\mathfrak{g} / \mathfrak{p}$ (uniquely) such that the associated invariant measure $d \bar{k}$ on $\left(M_{P} \cap K\right) \backslash K$ is normalized. Furthermore, let $d \bar{h}$ be the invariant measure on $(H \cap P) \backslash H$ determined by Lemma 5.1 with $L=H$.

Corollary 5.2 For every $f \in C\left(G: P: 1 \otimes 2 \rho_{P} \otimes 1\right)$ with $\operatorname{supp} f \subset P H$, we have

$$
\int_{\left(K \cap M_{P}\right) \backslash K} f(k) d \bar{k}=\int_{(H \cap P) \backslash H} f(h) d \bar{h} .
$$

Proof. By application of Lemma 5.1, both integrals equal the integral of the density $j_{\omega}(f)$ over $P \backslash G$.

Remark 5.3 The above result is due to [18, Lemma 1.3. Our proof is more conceptual.

Proof of Lemma 4.4. It suffices to prove an estimate similar to (4.5) for each of the finitely many components $\mathrm{ev}_{w} g$ of ev $g$, for $w \in \mathcal{W}$. Let $R_{w}$ denote the topological linear automorphism of $C^{-\infty}(K: \xi)$ given by $R_{w} g(k)=g(k w)$. Then $R_{w}$ maps $C^{-s}(K: \xi)$ isomorphically to itself for each $s>0$, and it restricts to a bijection

$$
C^{-\infty}(K: \xi)_{P, \lambda}^{H} \stackrel{\simeq}{\longrightarrow} C^{-\infty}(K: \xi)_{P, \lambda}^{w H w^{-1}} .
$$

Since $\mathrm{ev}_{e} \circ R_{w}=\mathrm{ev}_{w}$, it suffices to prove the estimate (4.5) with $\mathrm{ev}_{e}$ in place of ev.

Fix an element $v \in \mathcal{H}_{\xi}^{M \cap H}$. Then we shall complete the proof by establishing the existence of $C>0$ such that

$$
\left|\langle g(e) \mid v\rangle_{\xi}\right| \leq C(1+|\lambda|)^{s}\|g\|_{-s}
$$

where $\langle\cdot \mid \cdot\rangle_{\xi}$ denotes the unitary structure on $\mathcal{H}_{\xi}$. Fix a non-trivial and nonnegative compactly supported smooth function $\psi$ on $(H \cap P) \backslash H$. Define, for each $\lambda \in \mathfrak{a}_{\mathrm{qC}}^{*}$, an $\mathcal{H}_{\xi}$-valued function $\varphi_{\lambda}$ on $G$ as follows:

$$
\varphi_{\lambda}(x)= \begin{cases}a^{-\bar{\lambda}+\rho} \psi(h) \xi(m) v & \text { if } x=\operatorname{manh} \in P H, \\ 0 & \text { otherwise }\end{cases}
$$

where $m \in M, a \in A_{\mathrm{q}}, n \in N_{P}, h \in H$. Then, since $\psi$ is compactly supported, the support of $\varphi_{\lambda}$ is a closed subset of the open subset $P H$ of $G$, and hence 
$\varphi_{\lambda} \in C^{\infty}(P: \xi:-\bar{\lambda})$ (cf. [15], Thm. II.3.3). We claim that, with respect to the sesquilinear pairing (3.5),

$$
\left\langle g \mid \varphi_{\lambda}\right\rangle=\langle g(e) \mid v\rangle_{\xi} \int_{(H \cap P) \backslash H} \psi(h) d \bar{h}
$$

for all $g \in C^{-\infty}(P: \xi: \lambda)^{H}$. Indeed, since $g$ is smooth on $P H$, which contains the support of $\varphi_{\lambda}$, we have

$$
\left\langle g \mid \varphi_{\lambda}\right\rangle=\int_{K}\left\langle g(k) \mid \varphi_{\lambda}(k)\right\rangle_{\xi} d k
$$

and by Corollary 5.2, the integral over $K$ can be rewritten as the following integral over $(H \cap P) \backslash H$ :

$$
\int_{(H \cap P) \backslash H}\left\langle g(h) \mid \varphi_{\lambda}(h)\right\rangle_{\xi} d \bar{h} .
$$

Since $g(h)=g(e)$ and $\varphi_{\lambda}(h)=\psi(h) v$, the claimed formula (5.3) follows.

The integral in (5.3) is positive. Applying the inequality (3.6) to the left side of (5.3) we infer that

$$
\left|\langle g(e) \mid v\rangle_{\xi}\right| \leq C\|g\|_{-s}\left\|\varphi_{\lambda}\right\|_{s}
$$

for some constant $C$. We will finish the proof of (5.1) by showing that given $R \in \mathbb{R}$ and $s \in \mathbb{N}$, there exists $C>0$ such that

$$
\left\|\varphi_{\lambda}\right\|_{s} \leq C(1+|\lambda|)^{s}
$$

for all $\lambda \in \mathfrak{a}_{\mathrm{q}}^{*}(\bar{P}, R)$.

Fix a compact set $\Omega \subset H$ whose image contains supp $\psi$ under the mapping $h \mapsto(H \cap P) h, H \rightarrow(H \cap P) \backslash H$. Then $\operatorname{supp} \varphi_{\lambda} \subset P \Omega$ for all $\lambda$. If $\mathfrak{b}$ is a Lie algebra, and $s \in \mathbb{N}$, then by $U(\mathfrak{b})_{s}$ we denote the subspace of elements in $U(\mathfrak{b})$ of order at most $s$. The norm $\left\|\varphi_{\lambda}\right\|_{s}$ is dominated by a positive constant times the maximum of $\sup _{k \in K}\left\|R_{u} \varphi_{\lambda}(k)\right\|_{\xi}$ for $u$ ranging over a finite subset $S$ of $U(\mathfrak{k})_{s}$ (see (1.9)). Taking into account that $-\mathfrak{a}_{\mathrm{q}}^{*}(P, R)=\mathfrak{a}_{\mathrm{q}}^{*}(\bar{P}, R)$, we see from Lemma 5.4 below that we may as well estimate the norm $\sup _{h \in \Omega}\left\|\left(\pi_{P, \xi,-\bar{\lambda}}(u) \varphi_{\lambda}\right)(h)\right\|_{\xi}$ for each $u \in S$. The estimate (5.4) now follows by application of Lemma 5.5, also given below.

Lemma 5.4 Let $P \in \mathcal{P}_{\sigma}^{\min }$ and $\xi \in \widehat{M}_{\mathrm{fu}}$. Let $\Omega \subset H$ be a compact set, and $R \in \mathbb{R}$. There exists a constant $C>0$ such that

$$
\sup _{k \in K}\|\phi(k)\|_{\xi} \leq C \sup _{h \in \Omega}\|\phi(h)\|_{\xi},
$$

for all $\lambda \in \mathfrak{a}_{\mathrm{q}}^{*}(P, R)$ and all $\phi \in C(P: \xi: \lambda)$ with $\operatorname{supp} \phi \subset P \Omega$.

Proof. We first give the proof under the assumption that $H$ is connected. For $x \in P H$ we write $x=n(x) a(x) m(x) h(x)$ with $n(x) \in N_{P}, a(x) \in A_{\mathrm{q}}$, $m(x) \in M$ and $h(x) \in H$. The element $a(x)$ is unique and depends continuously 
on $x$. Moreover, we may arrange that $h(x) \in \Omega$ for $x \in P \Omega$. Then $\phi(k)=$ $a(k)^{\lambda+\rho} \xi(m(k)) \phi(h(k))$ for $k \in K \cap P H$. As $\xi$ is a unitary representation of $M$, it suffices to prove that $a(k)^{\lambda+\rho}$ is uniformly bounded for $k \in K \cap P \Omega$ and $\lambda \in \mathfrak{a}_{\mathrm{q}}^{*}(P, R)$.

For each $\alpha \in \Sigma$, let $H_{\alpha} \in \mathfrak{a}_{\mathrm{q}}$ be determined by $\nu\left(H_{\alpha}\right)=\langle\nu, \alpha\rangle$ for all $\nu \in \mathfrak{a}_{\mathrm{q}}^{*}$. It follows from [1], Thm. 3.8, that, for all $k \in K \cap P H$, the element $\log a(k)$ belongs to the closed cone spanned by the vectors $H_{\alpha}$, for $\alpha \in \Sigma(P)$. Moreover, by continuity and compactness, $\log a(k)$ belongs to a bounded subset of this cone, for all $k \in K \cap P \Omega$. Hence, there exist $c>0$ and numbers $r_{\alpha}(k) \in[0, c]$ such that $\log a(k)=\sum_{\alpha \in \Sigma(P)} r_{\alpha}(k) H_{\alpha}$ for all $k \in K \cap P \Omega$. It follows that

$$
(\operatorname{Re} \lambda+\rho)(\log a(k))=\sum_{\alpha \in \Sigma(P)} r_{\alpha}(k)\langle\operatorname{Re} \lambda+\rho, \alpha\rangle
$$

is bounded from above, uniformly for $k \in K \cap P \Omega$ and $\lambda \in \mathfrak{a}_{\mathrm{q}}^{*}(P, R)$.

It remains to treat the case where $H$ is not connected. Let $H_{e}$ denote the identity component of $H$. Then $H=H_{e}(K \cap H)$. Hence, $\Omega$ can be written as a disjoint union $\Omega_{1} k_{1} \cup \ldots \cup \Omega_{m} k_{m}$, where $k_{i} \in K \cap H$, and where $\Omega_{i} \subset H_{e}$ is compact, for $i=1, \ldots, m$. Without loss of generality we may assume that $\operatorname{supp} \phi \subset P \Omega_{i} k_{i}$ for some $i$, and then the result follows by application of the above to the right translate of $\phi$ by $k_{i}^{-1}$.

Lemma 5.5 Let $P \in \mathcal{P}_{\sigma}^{\min }$ and $\xi \in \widehat{M}_{\mathrm{fu}}$. Let $\varphi_{\lambda} \in C^{\infty}(P: \xi: \lambda)$ be a family of functions, for $\lambda \in \mathfrak{a}_{\mathrm{qC}}^{*}$, such that $\left.\varphi_{\lambda}\right|_{H}$ is independent of $\lambda$ and has support inside a set of the form $(H \cap M) \Omega$, with $\Omega \subset H$ compact. Then for every $s \in \mathbb{N}$ and every $u \in U(\mathfrak{g})$ of order at most $s$ there exists a constant $C>0$ such that

$$
\sup _{h \in \Omega}\left\|\pi_{P, \xi, \lambda}(u) \varphi_{\lambda}(h)\right\|_{\xi} \leq C(1+|\lambda|)^{s},
$$

for all $\lambda \in \mathfrak{a}_{\mathrm{qC}}^{*}$.

Let $h \in \Omega$. Since $\mathfrak{g}=\operatorname{Lie}(P)+\mathfrak{h}$ it follows from the Poincaré-BirkhoffWitt theorem that $u \in U(\mathfrak{g})_{s}$ can be written as a finite sum of products of the form $u_{h}:=\operatorname{Ad} h^{-1}\left(u_{h}^{\prime}\right) u_{h}^{\prime \prime}$, where $u_{h}^{\prime} \in U(\operatorname{Lie}(P))_{s}$ and $u_{h}^{\prime \prime} \in U(\mathfrak{h})_{s}$. Moreover, since $\Omega$ is compact, the elements $u_{h}^{\prime}, u_{h}^{\prime \prime}$ can be chosen such that they belong to bounded subsets of $U(\operatorname{Lie}(P))_{s}$ and $U(\mathfrak{h})_{s}$, respectively, for all $h \in \Omega$.

For each element $u^{\prime} \in U(\operatorname{Lie}(P))_{s}$ there exists a constant $C^{\prime}>0$ such that

$$
\left\|\left(\xi \otimes\left(\lambda+\rho_{P}\right) \otimes 1\right)\left(u^{\prime}\right)\right\|_{\text {op }} \leq C^{\prime}(1+|\lambda|)^{s},
$$

where $\|\cdot\|_{\text {op }}$ indicates the operator norm on $\operatorname{End}\left(\mathcal{H}_{\xi}\right)$. Moreover, the constant $C^{\prime}$ can be taken uniform if $u^{\prime}$ varies in a bounded subset of $U(\operatorname{Lie}(\mathrm{P}))_{s}$.

We apply this with $u^{\prime}=u_{h}^{\prime}$. Then

$$
\left\|\pi_{P, \xi, \lambda}\left(u_{h}\right) \varphi_{\lambda}(h)\right\|_{\xi} \leq C^{\prime}(1+|\lambda|)^{s}\left\|\pi_{P, \xi, \lambda}\left(u_{h}^{\prime \prime}\right) \varphi_{\lambda}(h)\right\|_{\xi} .
$$

It follows from the hypotheses that $\left\|\left(\pi_{P, \xi, \lambda}\left(u_{h}^{\prime \prime}\right) \varphi_{\lambda}\right)(h)\right\|_{\xi}$ is independent of $\lambda$ and uniformly bounded with respect to $h \in \Omega$. The estimate (5.5) now follows. 


\section{References}

[1] E. P. van den Ban. A convexity theorem for semisimple symmetric spaces. Pacific J. Math., 124(1):21-55, 1986.

[2] E. P. van den Ban. The principal series for a reductive symmetric space. I. $H$-fixed distribution vectors. Ann. Sci. École Norm. Sup. (4), 21(3):359$412,1988$.

[3] E. P. van den Ban. The principal series for a reductive symmetric space.

II. Eisenstein integrals. J. Funct. Anal., 109(2):331-441, 1992.

[4] E. P. van den Ban and H. Schlichtkrull. Fourier transform on a semisimple symmetric space. Invent. Math., 130(3):517-574, 1997.

[5] E. P. van den Ban and H. Schlichtkrull. The most continuous part of the Plancherel decomposition for a reductive symmetric space. Ann. of Math. (2), 145(2):267-364, 1997.

[6] E. P. van den Ban and H. Schlichtkrull. Fourier inversion on a reductive symmetric space. Acta Math., 182(1):25-85, 1999.

[7] E. P. van den Ban and H. Schlichtkrull. The Plancherel decomposition for a reductive symmetric space. I. Spherical functions. Invent. Math., 161(3):453-566, 2005.

[8] E. P. van den Ban and H. Schlichtkrull. The Plancherel decomposition for a reductive symmetric space. II. Representation theory. Invent. Math., 161(3):567-628, 2005.

[9] E. P. van den Ban and H. Schlichtkrull. A Paley-Wiener theorem for reductive symmetric spaces. Ann. of Math. (2), 164(3):879-909, 2006.

[10] A. Borel and N. R. Wallach. Continuous cohomology, discrete subgroups, and representations of reductive groups, volume 94 of Annals of Mathematics Studies. Princeton University Press, Princeton, N.J., 1980.

[11] P. Delorme. Formule de Plancherel pour les espaces symétriques réductifs. Ann. of Math. (2), 147(2):417-452, 1998.

[12] P. Delorme. Harmonic analysis on real reductive symmetric spaces. In Proceedings of the International Congress of Mathematicians, Vol. II (Beijing, 2002), pages 545-554, Beijing, 2002. Higher Ed. Press.

[13] M. Flensted-Jensen. Discrete series for semisimple symmetric spaces. Ann. of Math. (2), 111(2):253-311, 1980.

[14] Harish-Chandra. Harmonic analysis on real reductive groups. III. The Maass-Selberg relations and the Plancherel formula. Ann. of Math. (2), 104(1):117-201, 1976. 
[15] G. Heckman and H. Schlichtkrull. Harmonic analysis and special functions on symmetric spaces, volume 16 of Perspectives in Mathematics. Academic Press Inc., San Diego, CA, 1994.

[16] S. Helgason. Groups and geometric analysis, volume 113 of Pure and Applied Mathematics. Academic Press Inc., Orlando, FL, 1984. Integral geometry, invariant differential operators, and spherical functions.

[17] A. W. Knapp and E. M. Stein. Intertwining operators for semisimple groups. II. Invent. Math., 60(1):9-84, 1980.

[18] G. Ólafsson. Fourier and Poisson transformation associated to a semisimple symmetric space. Invent. Math., 90(3):605-629, 1987.

[19] T. Ōshima and T. Matsuki. A description of discrete series for semisimple symmetric spaces. In Group representations and systems of differential equations (Tokyo, 1982), volume 4 of Adv. Stud. Pure Math., pages 331390. North-Holland, Amsterdam, 1984.

[20] V. S. Varadarajan. Harmonic analysis on real reductive groups. Lecture Notes in Mathematics, Vol. 576. Springer-Verlag, Berlin, 1977.

[21] D. A. Vogan, Jr. and N. R. Wallach. Intertwining operators for real reductive groups. Adv. Math., 82(2):203-243, 1990.

[22] N. R. Wallach. On Harish-Chandra's generalized C-functions. Amer. J. Math., 97:386-403, 1975.

[23] N. R. Wallach. Real reductive groups. II, volume 132 of Pure and Applied Mathematics. Academic Press Inc., Boston, MA, 1992.

E. P. van den Ban

Mathematical Insitute

Utrecht University

PO Box 80010

3508 TA Utrecht

The Netherlands

E-mail: E.P.vandenBan@uu.nl
H. Schlichtkrull

Department of Mathematical Sciences

University of Copenhagen

Universitetsparken 5

2100 København $\varnothing$

Denmark

E-mail: schlicht@math.ku.dk 